\title{
Turbulence statistics in a fully developed channel flow submitted to a high temperature gradient
}

\author{
ADRIEN TOUTANT AND FRANCOISE BATAILLE \\ ${ }^{1}$ PROMES-CNRS, UPR 8521, Perpignan France \\ University of Perpignan Via Domitia \\ *Email: adrien.toutant@univ-perp.fr
}

(Received 12 July 2018)

Direct numerical simulations of a turbulent channel flow submitted to a high temperature gradient are performed. The temperatures of the channel walls are imposed to $293 \mathrm{~K}$ for the cold one and $586 \mathrm{~K}$ for the hot one. In this configuration, the flow is subsonic, while temperature variations can be strong and induce significant variations of the fluid properties. The unsteady low Mach number Navier-Stokes equations are solved numerically at a Reynolds number of 6400 , based on the mean centreline velocity, mean centreline viscosity and channel half-width. The mesh that contains about 470 millions of grid points allows to resolve all essential turbulence scales and no subgrid model is used. The independence of the mesh resolution is shown. A large number of turbulence statistics are computed. The profiles obtained at the cold and at the hot walls are compared using different non-dimensionalizations. The coupling between the turbulence and the temperature gradient is shown to modify the mean and the fluctuation profiles.

\section{Introduction}

Wall-bounded turbulent flows a very active area of research (Lagha et al. (2011); Marusic et al. (2011); Wei \& Pollard (2011)). Many industrial processes have temperature gradients in the near wall region (concentrated solar power tower as presented by Daguenet-Frick et al. (2012), nuclear power plants, piston engines, propulsion systems, heat exchangers ...). In the case of small temperature gradients, the turbulent boundary layer with heat transfer remains an incompressible flow (Morinishi et al. (2007); Wu \& Moin (2010)). In the situation of high temperature gradients, the interactions between the energy and the momentum equations are strong and the classical isothermal models are valid. The temperature gradient can be considered as a strong external agency that modify the turbulence properties (Serra et al. (2012c)). Turbulence modifications have been shown, for example, for strong magnetic field, rotation, or stratification (Mahalov et al. (1998); Ye \& Zhou (2010); Zhou \& Oughton (2011)). In the case of supersonic compressible flow, the coupling between turbulence and high temperature gradients has been studied extensively to increase the understanding of the turbulent boundary layer mechanism (Huang et al. (1995); Coleman et al. (1995); Morinishi et al. (2004); Tamano \& Morinishi (2006); Spina et al. (1994)). In the case of low speed flow, only very few studies are dedicated to this coupling. In particular, there is no reference data subsonic flow without low Reynolds number effect and with compressible effect due to strong thermal gradient. This case is missing in the literature. The present paper gives the results of a direct numerical simulation (DNS) that studies this very specific situation. The configuration is represented by figure 1. It is a bi-periodic channel flow with a cold wall 
and a hot one. The lengths of the periodic directions $(L x$ and $L z)$ are different according to litterature. The turbulent Reynolds number used in this table is defined by the mean Reynolds number of the cold (subscript 1) and the hot (subscript 2) walls:

$$
R e_{\tau m}=\frac{R e_{\tau 1}+R e_{\tau 2}}{2}
$$

The turbulent Reynolds number is based on the friction velocity $u_{\tau}=\sqrt{\frac{\tau_{w}}{\rho_{w}}}$ (where $\tau_{w}$ is the wall shear stress), the kinematic viscosity at the wall $\nu_{w}$ and the channel half-width $h$ :

$$
R e_{\tau}=\frac{u_{\tau} h}{\nu_{w}}
$$

The table 1, that presents the previous studies dedicated to fully developed channel flow, illustrates well the lack of reference data concerning high temperature gradients and high Reynolds number. Kim et al. (1987); Moser et al. (1999) realized direct numerical simulation (DNS) with a temperature ratio of 1 (the incompressible limit case). Debusschere \& Rutland (2004) made DNS of passive heat transfer in a plane channel and Couette flow. These simulations appear only for a temperature ratio of 1 in the table because they consider the temperature as a passive scalar (no dynamical effect). Kawamura et al. $(1999,2000)$ realized DNS with different heat flux at the wall. Again, these simulations appear only for a temperature ratio of 1 in the table because the fluid properties are supposed constant (temperature is a passive scalar).

Lessani \& Papalexandris (2006, 2007) Wang \& Pletcher (1996) Nicoud (1998) realized the only DNS in this configuration and with a significant temperature ratio. He considered temperature ratios of 1.01, 2 and 4 with two kinds of law for the property variations. The mean and the turbulent profiles are asymmetric even with semi-local scalings. Nevertheless, these studies consider only a low mean turbulent Reynolds number $\left(R e_{\tau m}=180\right)$. Consequently, it is impossible to be sure that the observations are due to the temperature gradient or to a low Reynolds number effect. Indeed, in Nicoud (1998), the turbulent Reynolds number at the hot side is $R e_{\tau 2}=82$ and the author makes the hypothesis that some of the obtained results may be due to a low Reynolds number effect. In our previous work (Serra et al. $(2012 a, b, c)$ ), we realized LES parametric studies for different temperature ratios and turbulent Reynolds numbers. It allowed to investigate the influence of the variations of the viscosity and the conductivity, the effect of the choice of thermal subgrid-scale models and the modifications induced by a rise in the temperature ratio. We observed that when the temperature ratio increases, the profiles become asymmetric. Moreover, the levels and the locations of the peaks of the fluctuations and correlations are modified. We are very confident of the tendency of the obtained LES results. However, it is required to realize DNS for quantify precisely the different peak levels.

The objective of the present work is to generate data which are not available for low speed flow with large temperature gradient and without low Reynolds number effect. The mean turbulent Reynolds number of the present study is $R e_{\tau m}=400$ with at the cold side $R e_{\tau 1}=565$ and at the hot side $R e_{\tau 2}=235$. It corresponds to a Reynolds number of 6400 , based on the mean centreline velocity, mean centreline viscosity and channel half-width. The numerical method is described in section 2 . The section 3 is dedicated to the domain size and the grid spacing of the mesh. Statistics are calculated and discussed in section 4 . 


\begin{tabular}{||c||c||c||}
\hline$\frac{T_{2}}{T_{1}}$ & $R e_{\tau m}=180$ & $R e_{\tau m}=395$ \\
\hline 1 & Kim et al. (1987) & Moser et al. $(1999)$ \\
& Debusschere \& Rutland (2004) & $\begin{array}{l}\text { Kawamura } \text { et al. }(1999) \\
\text { Kawamura } \text { et al. }(2000)\end{array}$ \\
\hline 1,01 & Nicoud (1998) & \\
\hline 2 & Nicoud (1998) & \\
\hline
\end{tabular}

TABLE 1 . in fully developed channel flow with variable properties. $R e_{\tau m}$ is the mean turbulent Reynolds number. $T_{2}$ is the temperature of the hot wall and $T_{1}$ the temperature of the cold wall.

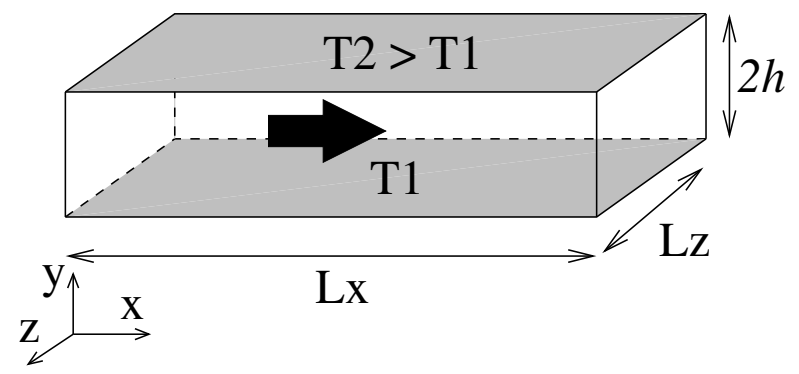

Figure 1. Computational domain

\section{Numerical method}

A low Mach number approximation is applied to the 3D Navier-Stokes equations (see Paolucci (1982); Majda \& Sethian (1985)). This decouples the density from the pressure and avoids the acoustics and the corresponding CFL restriction on the time step. In the case of an ideal gas without volume force (the gravity is negligible) and heat source, the low Mach number equations are the following.

- Conservation of mass equation

$$
\frac{\partial \rho}{\partial t}+\frac{\partial\left(\rho U_{j}\right)}{\partial x_{j}}=0
$$

- Conservation of momentum equation

$$
\rho \frac{\partial U_{i}}{\partial t}+\rho U_{j} \frac{\partial U_{i}}{\partial x_{j}}=-\frac{\partial P_{d y n}}{\partial x_{i}}+\frac{\partial}{\partial x_{j}}\left[\mu\left(\frac{\partial U_{i}}{\partial x_{j}}+\frac{\partial U_{j}}{\partial x_{i}}\right)\right]-\frac{2}{3} \frac{\partial}{\partial x_{i}}\left(\mu \frac{\partial U_{j}}{\partial x_{j}}\right)
$$

- Conservation of energy equation

$$
\rho C_{p}\left(\frac{\partial T}{\partial t}+U_{j} \frac{\partial T}{\partial x_{j}}\right)=\frac{\partial P_{\text {thermo }}}{\partial t}+\frac{\partial}{\partial x_{j}}\left(\lambda \frac{\partial T}{\partial x_{j}}\right)
$$

- Equation of state

$$
P_{\text {thermo }}=r \rho T
$$

- The thermodynamic pressure is a spatial constant

$$
\frac{\partial P_{\text {thermo }}}{\partial x_{i}}=0
$$

In this set of equations, $\rho$ is the density, $U_{i}$ are the velocity components, $T$ is the temperature, $t$ represents the time, $x_{i}$ are the coordinates, $\lambda$ and $\mu$ are the conductivity and the dynamic viscosity, $C_{p}$ is the constant pressure heat capacity, $P_{d y n}$ is the dynamic pres- 
sure, $P_{\text {thermo }}$ is the thermodynamic pressure and $r$ is the ideal gas specific constant. The gas considered in this study is air. The ideal gas specific constant is $r=287 \mathrm{~J} \cdot \mathrm{kg}^{-1} \cdot \mathrm{K}^{-1}$. We chose a Prandtl number $\operatorname{Pr}$ equal to 0.71 . We assume that the constant pressure heat capacity is constant: $C_{p}=1005 \mathrm{~J}_{\mathrm{kg}} \mathrm{g}^{-1} \cdot \mathrm{K}^{-1}$. At the beginning of the simulation, the thermodynamic pressure is $P_{\text {thermo }}=10^{5} \mathrm{~Pa}$. The conductivity $\lambda$ and the dynamic viscosity $\mu$ are solved using Sutherland law:

$$
\begin{gathered}
\mu(T)=1.461 .10^{-6} \frac{T^{1.5}}{T+111} \\
\lambda(T)=\frac{\mu C p}{P r}=\frac{1.468 .10^{-3}}{\operatorname{Pr}} \frac{T^{1.5}}{T+111}
\end{gathered}
$$

The low Mach number equations are solved in a fully developed non-isothermal channel flow (see figure 1). The DNS is carried out with the Trio_U code (Calvin et al. (2002)) developed at the French Atomic Agency . To solve a fully developed flow, we use a periodic boundary condition in the streamwise and spanwise directions. The DNS assumes a constant mass flux. For the wall boundary condition, we use constant temperature and no slip condition. The temperature at the lower wall is $T_{1}=293 \mathrm{~K}$ and at the upper wall $T_{2}=586 \mathrm{~K}$. Time integration is carried out by a third order Runge-Kutta scheme. The convection scheme for the velocity is a second order centered scheme (Brillant et al. (2005)). For the temperature, we use a third order quick scheme, as recommended by Châtelain et al. (2004) and Brillant et al. (2005). The numerical method used to solve the low Mach number equations is described in detail by Elmo \& Cioni (2003).

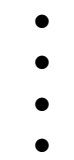

The mesh contains about 470 millions of grid points. At our knowledge, it is the biggest mesh for computational fluid dynamic with heat transfer. The simulation is realized in several cores. The mesh is partitioned in $32 \times 8 \times 16=4096$ sub-domains. Each sub-domain is attributed to a core and contains 115000 cells. The mesh is also realized in parallel. The projection step that calculates the dynamic pressure uses a parallel multigrid method. The multigrid method allows to divide by ten the time of this projection step. It uses 5 grid levels (4 agglomerations: 2 in y and 2 isotropic).

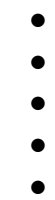

The coarse level is 100 times lighter than the fine level. In the five grid levels, seven multigrid V-cycles allow to divide the residual by a factor of 1010. We use a Jacobi smoother with 12 iterations of pre-smoothing and 12 iterations of post-smoothing. The preconditioned conjugate gradient with the symmetric successive over relaxation (SSOR) preconditioner that resolves the dynamic pressure on the coarse level grid represents 40 percent of the projection step.

The statistics of section 3 are realized by time integration. The statistics of section 4 are realized by time integration and by averaging over horizontal planes (homogeneous directions). The period of time integration for the turbulence statistics is about 3000 non-dimensional units of time after the flow reaches the fully developed state. The nondimensional unit of time is calculated at the hot wall $\nu_{2} /\left(u_{\tau 2}\right)^{2}$ (because it is bigger 
at the hot wall than at the cold one). This time period (3000 non-dimensional units of time) corresponds to 11 diffusion times $\left(h / u_{\tau m}\right.$ where $u_{\tau m}=\frac{u_{\tau 1}+u_{\tau 2}}{2}$ is the mean friction velocity). The statistically steady state is identified by the convergence of the root-mean-square velocity and temperature fluctuations.

\section{Computational domain and grid spacing}

The computational domain and the coordinate system are shown in figure 1. In a fully developed channel flow, the streamwise $(x)$ and the spanwise $(z)$ directions are homogeneous and periodic boundary conditions are applied. The size of the domain has to assure that the turbulence fluctuations are uncorrelated at the half of the channel. The streamwise and the spanwise lengths are chosen to be $L x=4 \pi h$ and $L z=2 \pi h$ where $2 h$ is the distance between the walls and $h=0.015 \mathrm{~m}$. The computation is carried out with $1537 \times 397 \times 769=469235341$ grid points for a temperature ratio $T_{r}=\frac{T_{2}}{T_{1}}=2$ and a Reynolds number of 6400 , based on mean centreline velocity, mean centreline viscosity and channel half-width. The mesh is non-uniform in the normal direction $(y)$ and uniform in the streamwise and spanwise directions. The grid points in the normal direction are determined by the following hyperbolic tangent transformation

$$
y_{k}=h\left\{1+\frac{1}{a} \tanh \left[\left(-1+2 \frac{k-1}{N_{y}-1}\right) \operatorname{atanh}(a)\right]\right\}, k \in\left[1, N_{y}\right]
$$

where $N_{y}=397$ is the number of grid points the $y$ axis and $a$ is a constant equal to 0.857745 . The non-dimensional grid spacings are scaled by wall variables

$$
\begin{aligned}
& y^{+}=\frac{y u_{\tau}}{\nu} \\
& u_{\tau}=\sqrt{\frac{\tau_{w}}{\rho_{w}}}
\end{aligned}
$$

where $\nu$ is the kinematic viscosity and $\tau_{w}$ the wall shear stress. Because the upper wall is two times warmer than the lower wall, the kinematic viscosity $\nu$ and the wall shear velocity $u_{\tau}$ are bigger at the upper wall. At the opposite, the wall shear velocity divided by the kinematic viscosity is bigger at the lower wall (the cold one). Consequently, we use the kinematic viscosity and the wall shear velocity of the cold wall to calculate the non-dimensional grid spacings. With this choice, we maximize the non-dimensional grid spacings and make sure that the grid resolution is sufficiently fine. The non-dimensional grid spacings in the streamwize and in the spanwise directions are the same:

$$
\Delta x^{+}=\Delta z^{+}=4.5
$$

In the normal direction, the first mesh point away from the wall is at $y^{+}=0.5$ and the maximum spacing at the centreline of the channel is 4.2 wall units.

Lele (1992)Kim et al. (1987)

Two-point correlations, mean velocity, velocity fluctuations and energy spectra are shown in figures 3 to 9 to demonstrate the correctness of the domain size and the grid resolution. The two-point correlations shown in figures 3 and 4 are calculated in the streamwise and in the spanwise directions at two distances from the wall (one very close and the other close to the centreline). Figure 3 concerns two-point velocity correlations and figure 4 two-point temperature correlations. The two-point velocity and temperature correlations fall off to zero values. Therefore, the computational domain is sufficiently large. To demonstrate the adequacy of the grid resolutions, we study the mesh convergence. Three simulations with different grid resolutions are realized: 


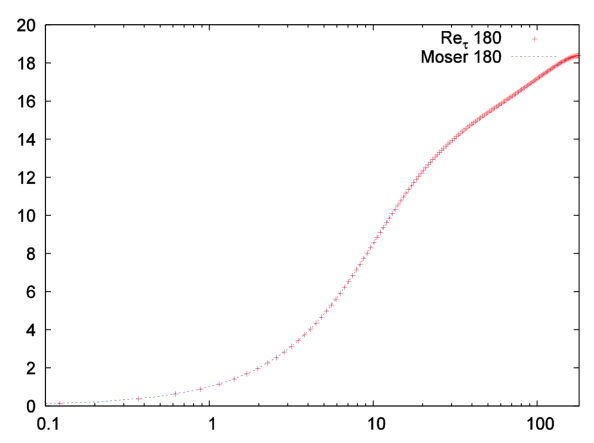

(a)

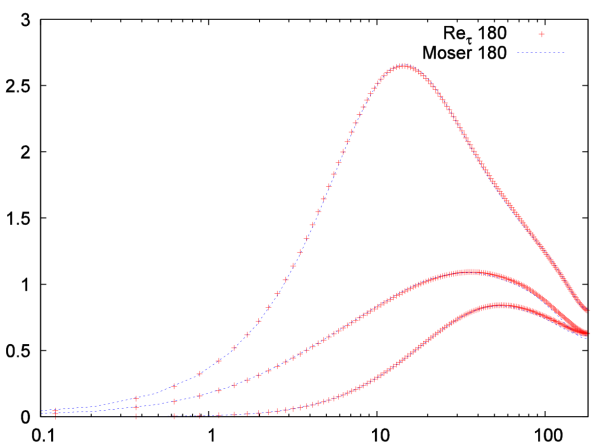

(b)

Figure 2. Kim et al. (1987).

- Rough mesh: 513x265x257,

- Fine mesh: $769 \times 397 \times 385$,

- Very fine mesh: $1537 \times 397 \times 769$.

The very fine mesh corresponds to the DNS studied in details in this contribution. Figure 5 shows the mean velocity profile for the three grid resolutions: the three profiles match . In figure 6 , the root-mean-square velocity fluctuations normalized by wall shear velocity of the cold side are plotted in global coordinates and wall coordinates. One can note small differencies between the rough mesh and the fine mesh and a very good adequacy between the fine and very fine meshes. The three profiles of the mean temperature for the three grid resolutions match perfectly figure 7 . The root-mean-square temperature fluctuations normalized by friction temperature of the cold side are plotted with global coordinates in figure 8. Although there are differencies between the rough and fine meshes, the profiles of the fine and very fine meshes are in very good agreement. Richardson (1911) :

Consequently, mesh convergence is obtained and the very fine mesh allows to resolve all essential turbulence scales. Finally, figure 9 represents the one-dimensional energy spectra for the fine and the very fine meshes. The shape of these spectra is the same for the two grid resolutions: the energy level and the slope of the spectra are identical. For the very fine mesh, the energy level associated with the high wavenumbers is six decades lower than the energy level associated to low wavenumbers. the smallest scales of the very fine mesh are not resolved by the fine mesh and the spectrum shapes are the same for the two grid resolutions, we can see that the dynamical roles of the non-resolved small scales is negligible. (Hinze (1975))

Figure 10 shows the one-dimensional temperature spectra. It illustrates again that the grid resolution is adequate since the level of temperature spectra associated with the high wavenumbers is five decades lower than the level of temperature spectra corresponding to low wavenumbers.

According these results, we can conclude that the domain size is large enough. We also see that the grid resolution is sufficient required to resolve all essential scales of turbulence for velocity and temperature. 

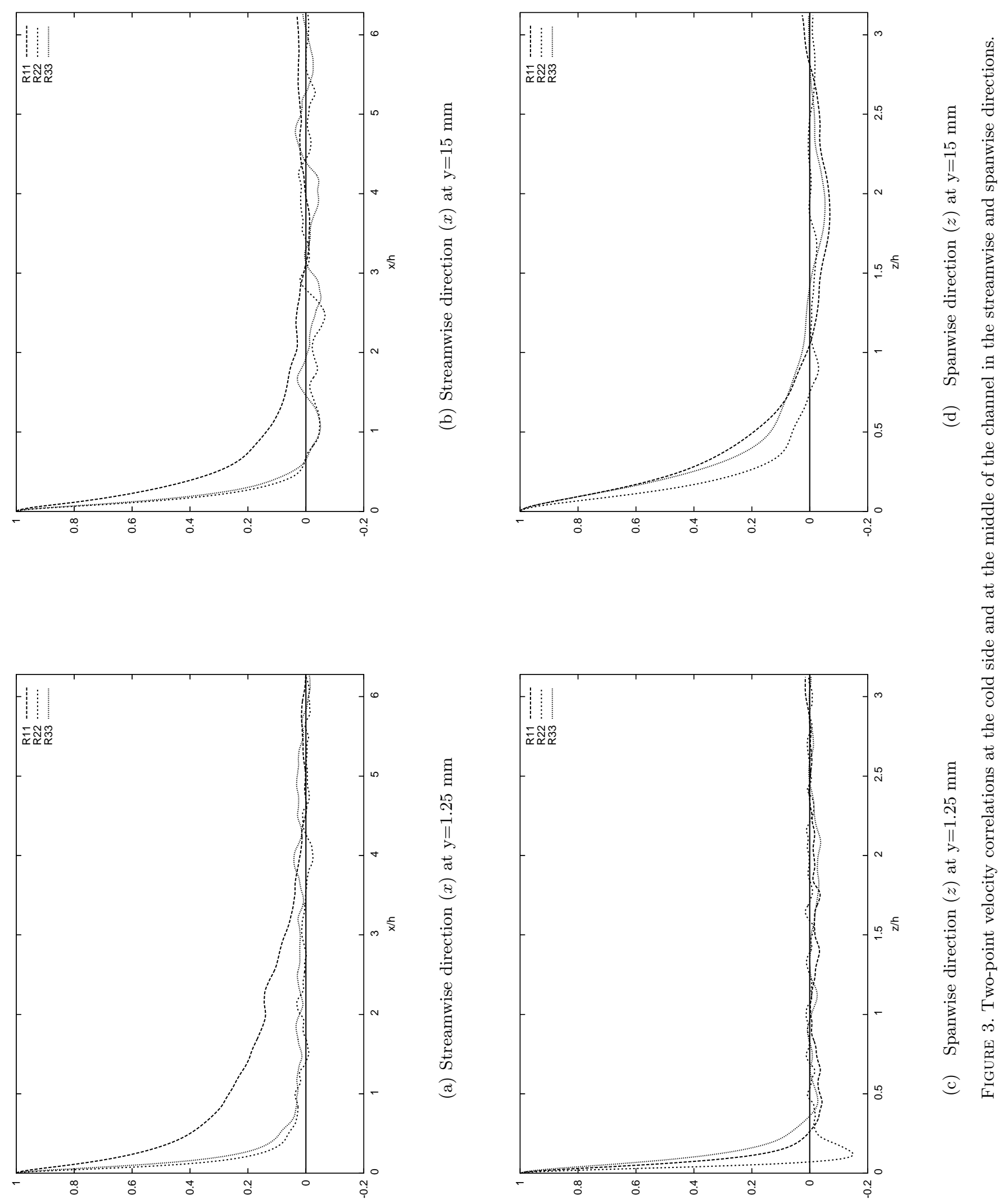


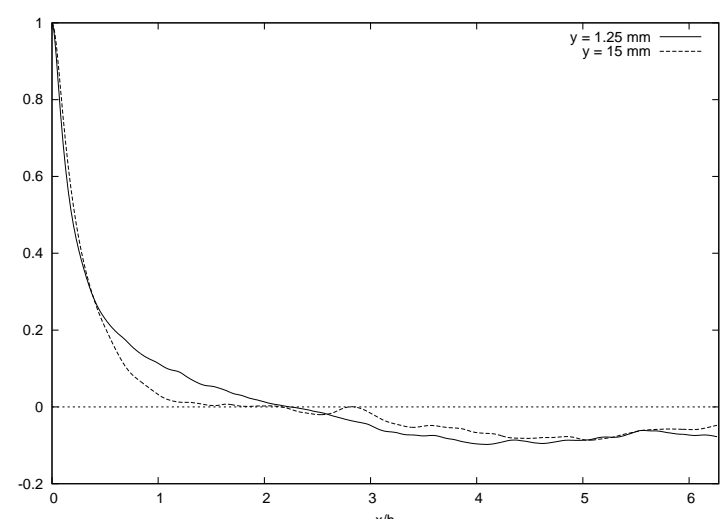

(a) Streamwise direction $(x)$ at $\mathrm{y}=1.25 \mathrm{~mm}$ and $\mathrm{y}=15 \mathrm{~mm}$

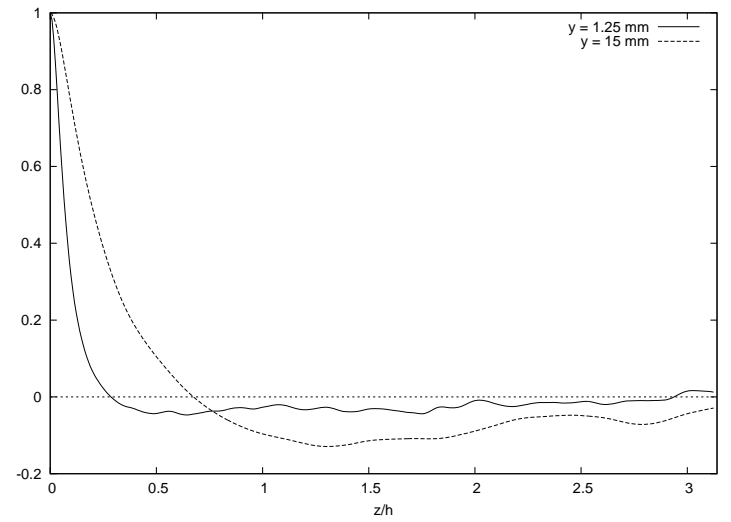

(b) Spanwise direction $(z)$ at $\mathrm{y}=1.25 \mathrm{~mm}$ and $\mathrm{y}=15 \mathrm{~mm}$

Figure 4. Two-point temperature correlations at the cold side and at the middle of the channel in the streamwise and spanwise directions.

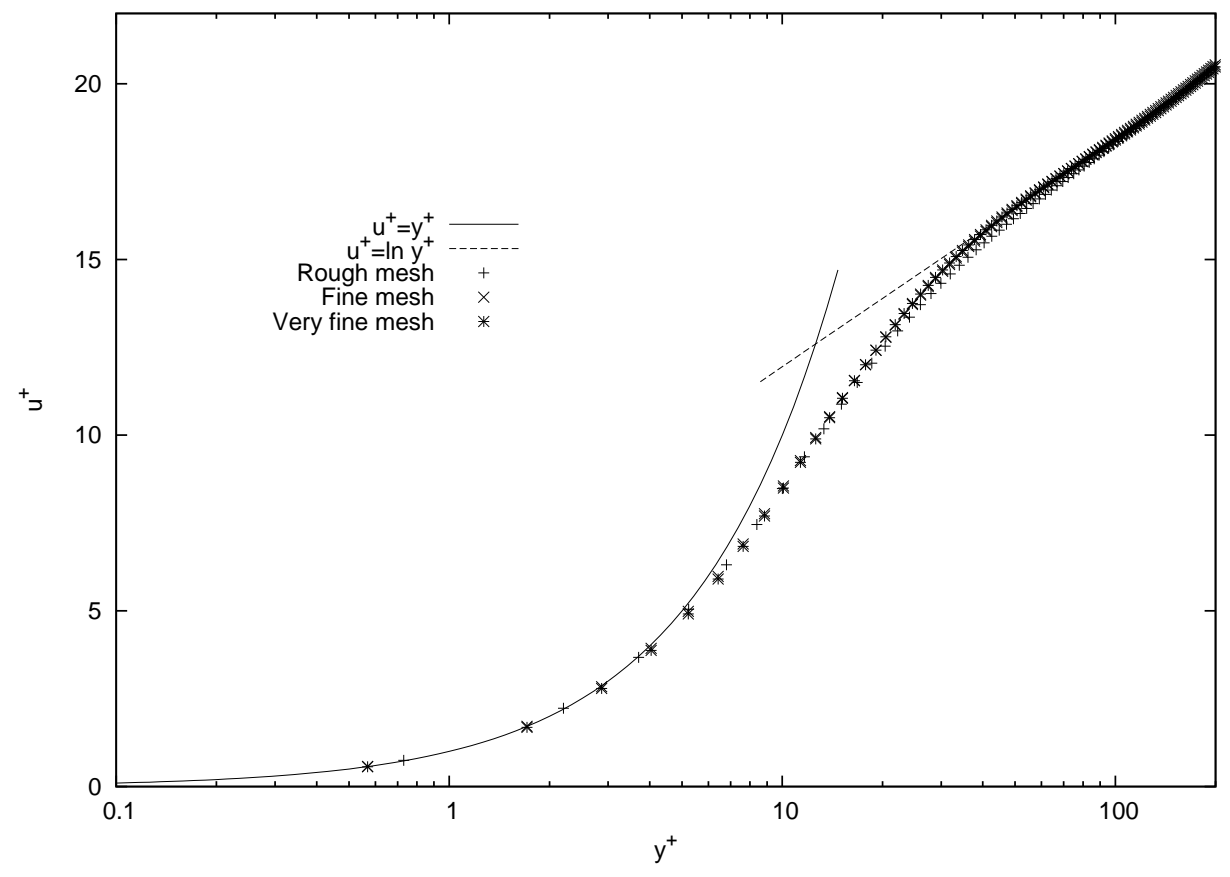

Figure 5. Mean velocity profiles at the cold wall. Comparison of the results obtained with three different meshes. 


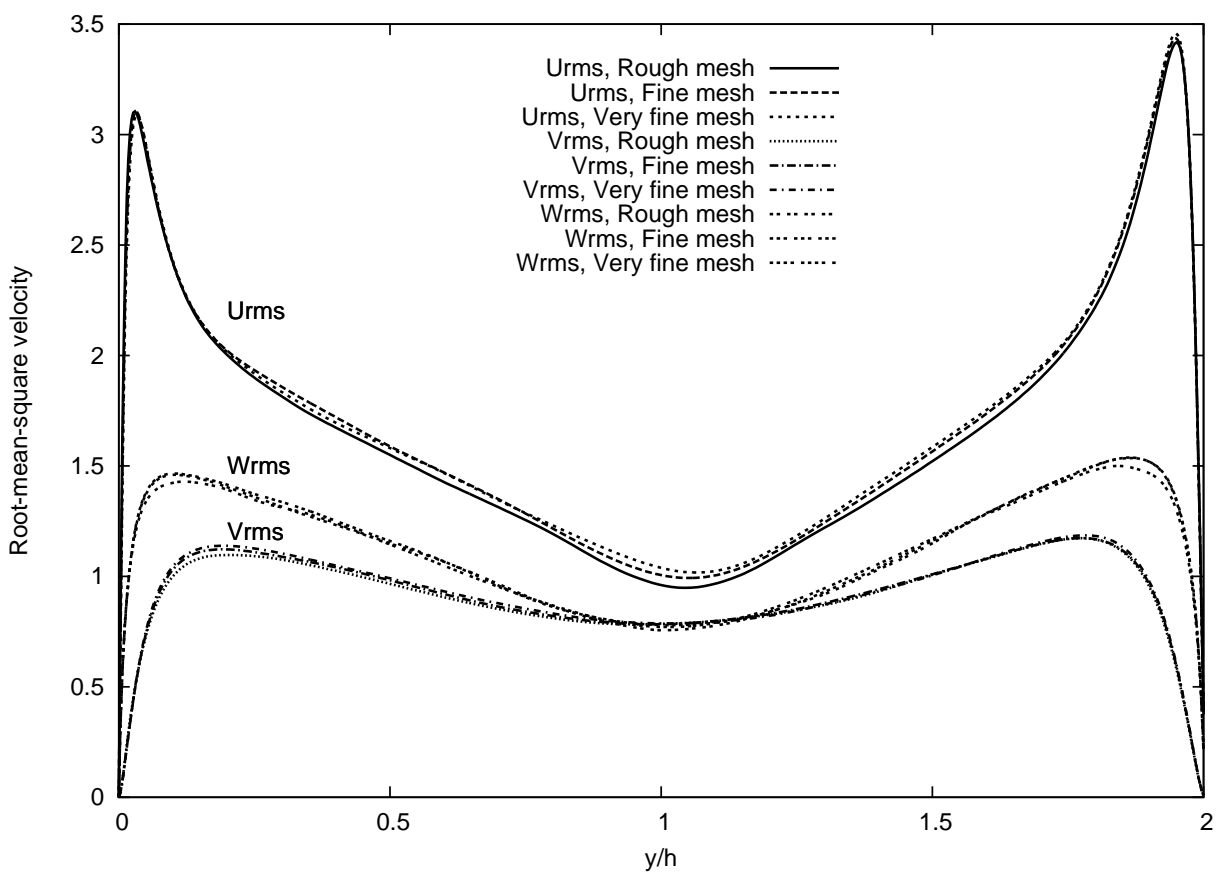

(a) Root-mean-square velocity in global coordinates

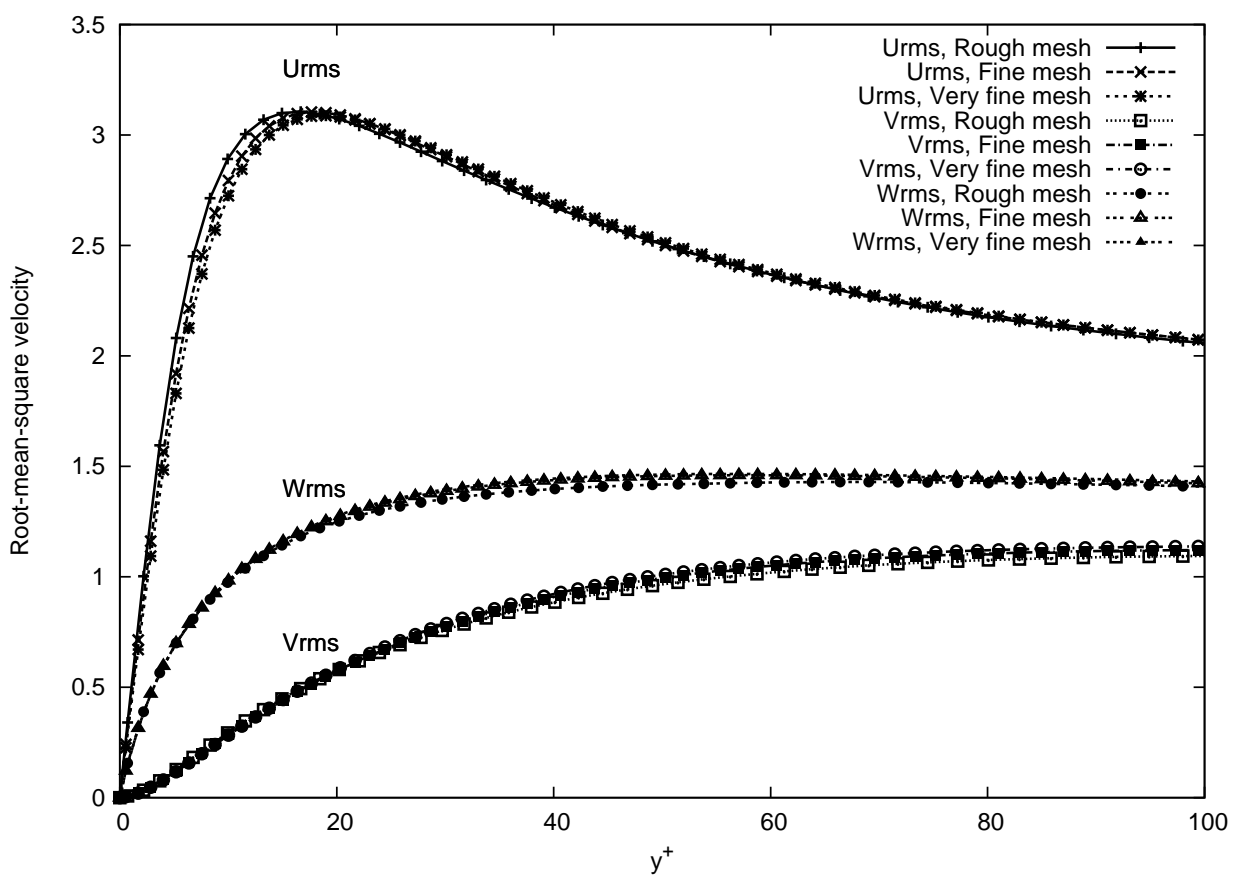

(b) Root-mean-square velocity at the cold side in wall coordinates

FIGURE 6. Root-mean-square velocity fluctuations normalized by wall shear velocity of the cold side in global coordinates and wall coordinates. Comparison of the results obtained with three different meshes. 


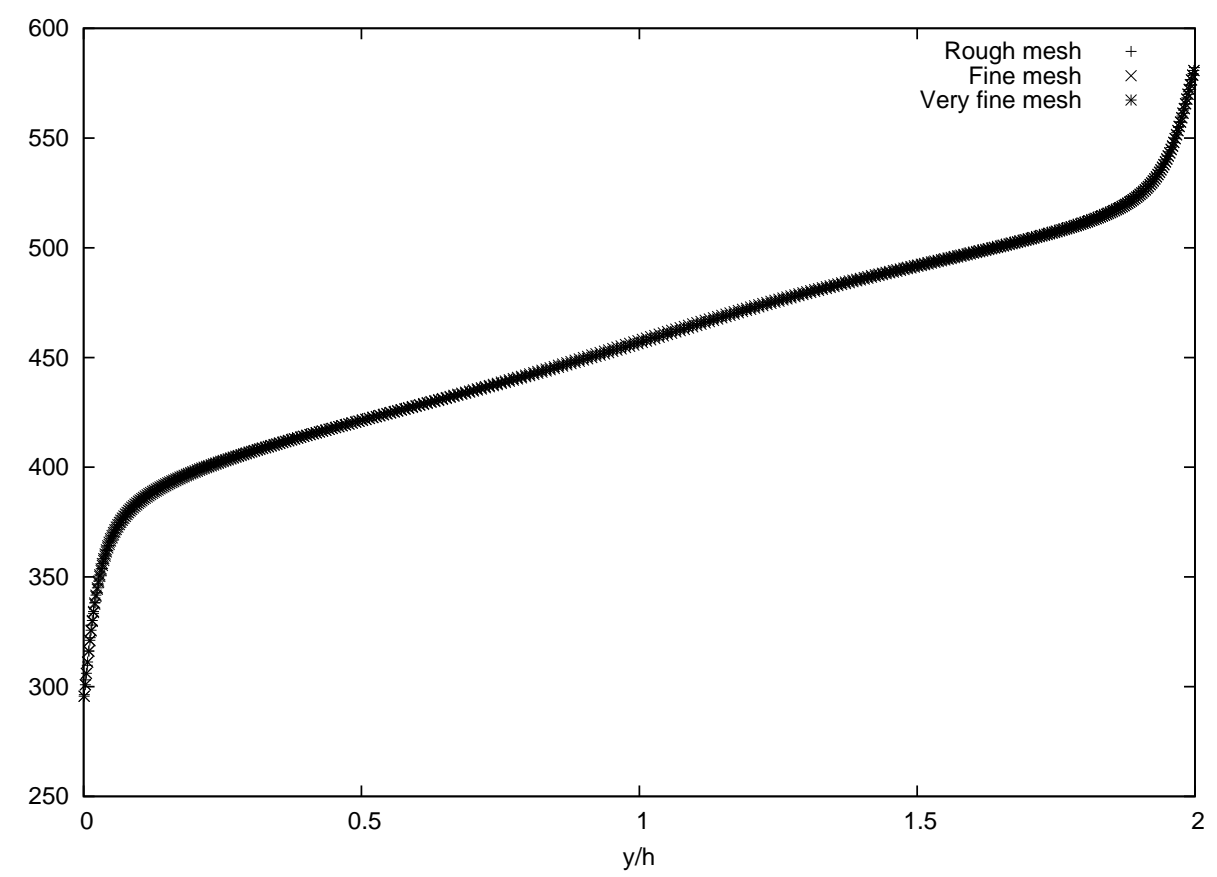

Figure 7. Mean temperature profiles. Comparison of the results obtained with three different meshes.

\section{Turbulence statistics}

We recall that in the present study the mean turbulence Reynolds number is $R e_{\tau m}=400$ with at the cold wall $R e_{\tau 1}=565$ and at the hot wall $R e_{\tau 2}=235$.

\subsection{Velocity}

In this section, some comparisons are done with the incompressible case at a turbulent Reynolds number $R e_{\tau}=395$. In the incompressible case, the fluid properties do not depend on the temperature and the turbulent Reynolds number are the same at the two walls $\left(R e_{\tau}=R e_{\tau m}=R e_{\tau 1}=R e_{\tau 2}\right)$. The data are provided by the database of Kawamura (2008). In figure 11, we can see in a semi-log plot that the mean velocity profile of the cold and hot walls scaled by the local friction velocity (the friction velocity is different at the cold and the hot walls) does not collapse. Furthermore, these profiles do not matched the classical law-of-the-wall:

$$
u^{+}=2.5 \ln \left(y^{+}\right)+5.5
$$

We observe that, at the hot wall, the additive constant is modified (4.5 instead of 5.5) and that, at the cold wall, the slope is increased by the temperature gradient (2.8 instead of 2.5). However, once transformed as proposed by Van Driest (1951)

$$
u^{+}=\frac{2}{P r_{t} T_{\tau} / T_{w}}\left(1-\sqrt{1-P r_{t} T_{\tau} / T_{w} u / u_{\tau}}\right)
$$




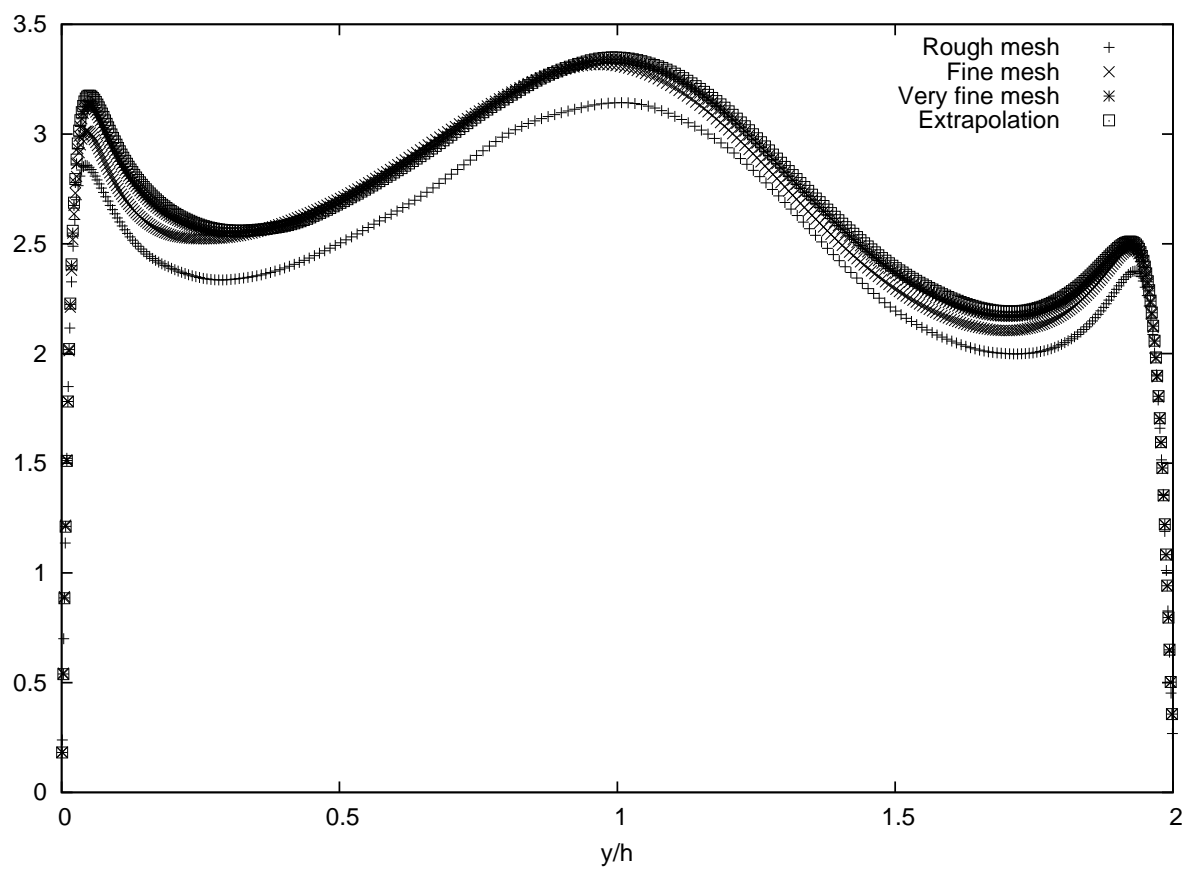

FIGURE 8. Root-mean-square temperature fluctuations normalized by friction temperature in global coordinates. Comparison of the results obtained with three different meshes Richardson (1911).

where $\operatorname{Pr}_{t}=0.9$ is the turbulent Prandtl number, $T_{\tau}$ the friction temperature (eq. 4.7 ) and $T_{w}$ the wall temperature, the cold and the hot profiles collapse perfectly (see figure 12). Moreover, the classical incompressible behavior is clearly obtained for the slope and the additive constant (see figure 12). This result shows that, the modification of the additive constant obtained by Nicoud (1998) seems to be due to low Reynolds effect. Nicoud \& Bradshaw (2000) underlined the fact that the Van Driest transformation is mathematically analogous to the Simpson transformation of the velocity profile for incompressible turbulent wall flows with uniform injection (Simpson (1970)). Identifying the two transformations, one that the equivalent transpiration velocity used in the Van Driest scaling is:

$$
V_{i n j}=\operatorname{Pr}_{t} T_{\tau} / T_{w} u_{\tau}
$$

Figure 13 shows that this equivalent transpiration velocity is a very good aproximation of the wall-normal velocity at the cold and the hot walls $\dagger$. Consequently, a possible mechanism of the interaction between the dynamic and thermal fields is the following:

- the temperature gradient creates a significant variation of density,

- the density variation creates a mean wall-normal velocity (mass conservation),

- the mean wall-normal velocity modifies the mean streamwise velocity.

$\dagger$ In the incompressible case, the mean wall-normal velocity is equal to zero. 


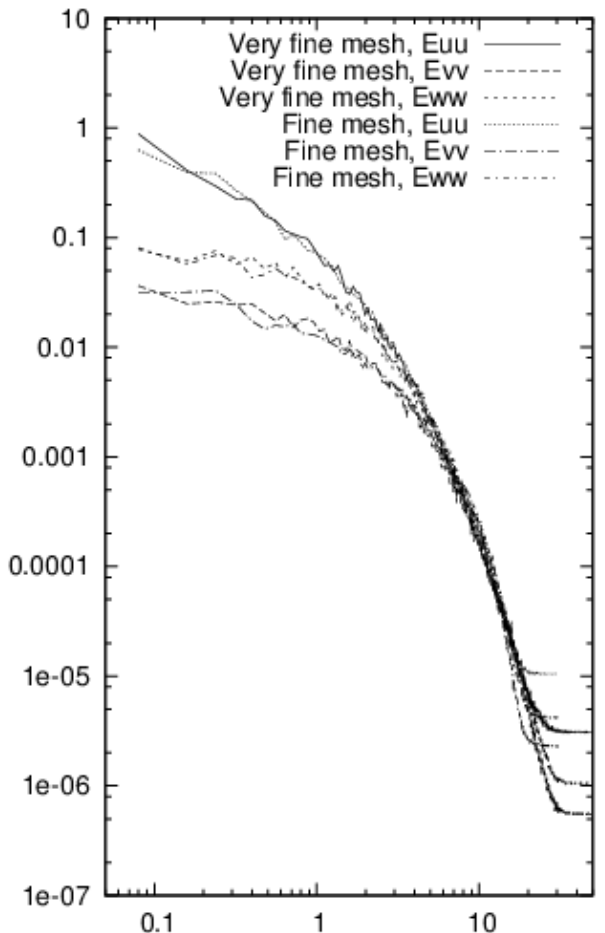

(a) Streamwise direction $\left(k_{x}\right)$ at $\mathrm{y}=1.25 \mathrm{~mm}$

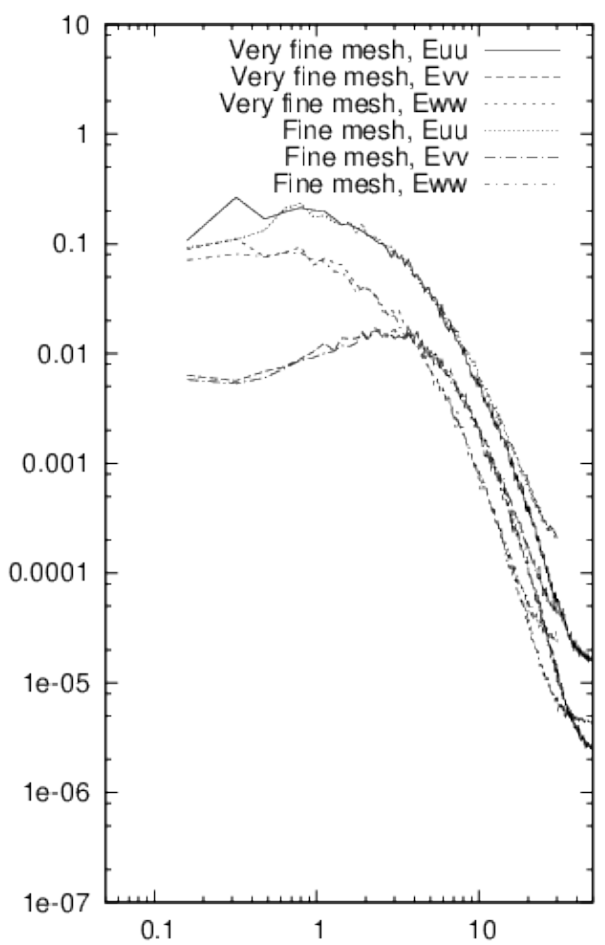

(c) Spanwise direction $\left(k_{z}\right)$ at $\mathrm{y}=1.25 \mathrm{~mm}$

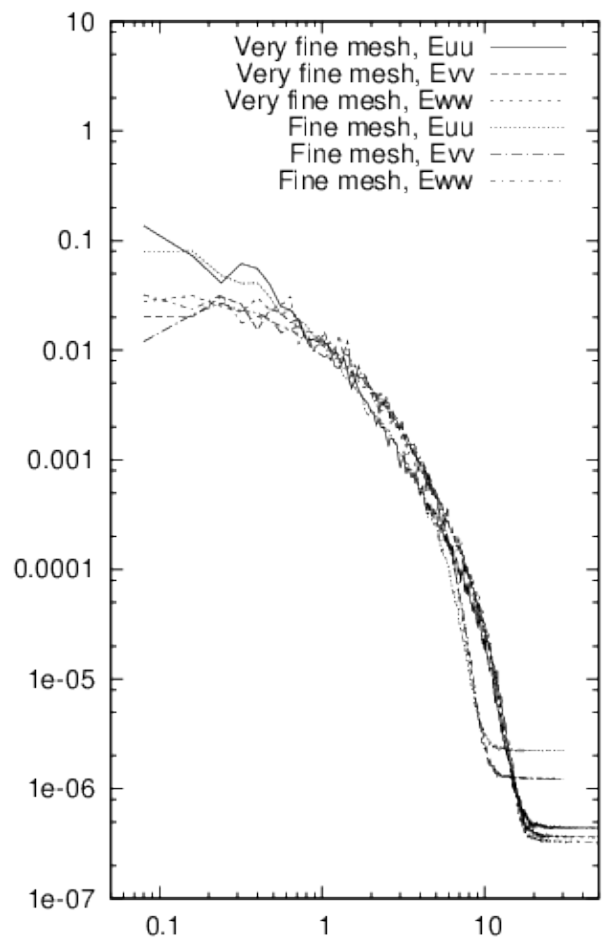

(b) Streamwise direction $\left(k_{x}\right)$ at $\mathrm{y}=15 \mathrm{~mm}$

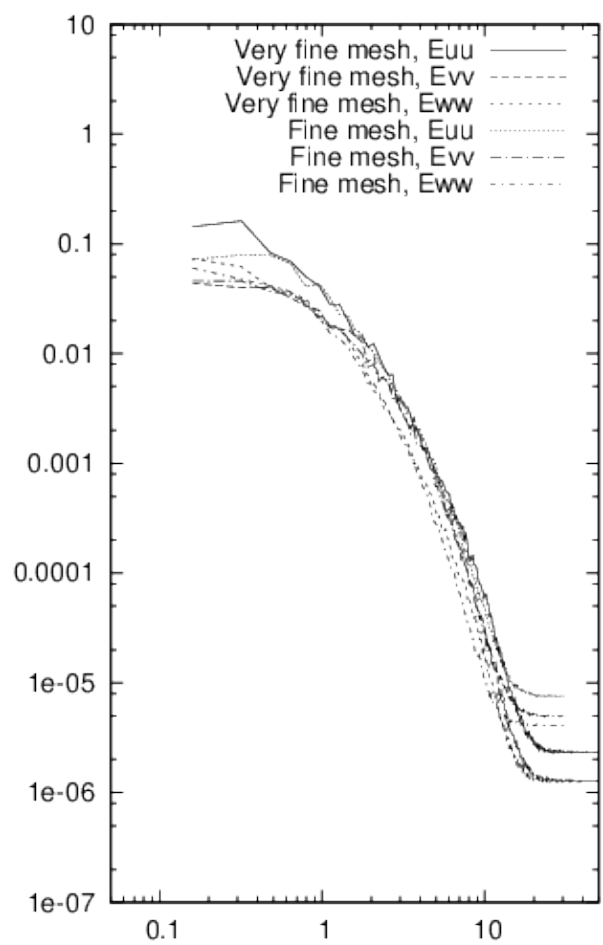

(d) Spanwise direction $\left(k_{z}\right)$ at $\mathrm{y}=15 \mathrm{~mm}$

FiguRE 9. One-dimensional energy spectra at the cold side and at the middle of the channel in the streamwise and spanwise directions.Comparison of the results obtained with two different meshes. 

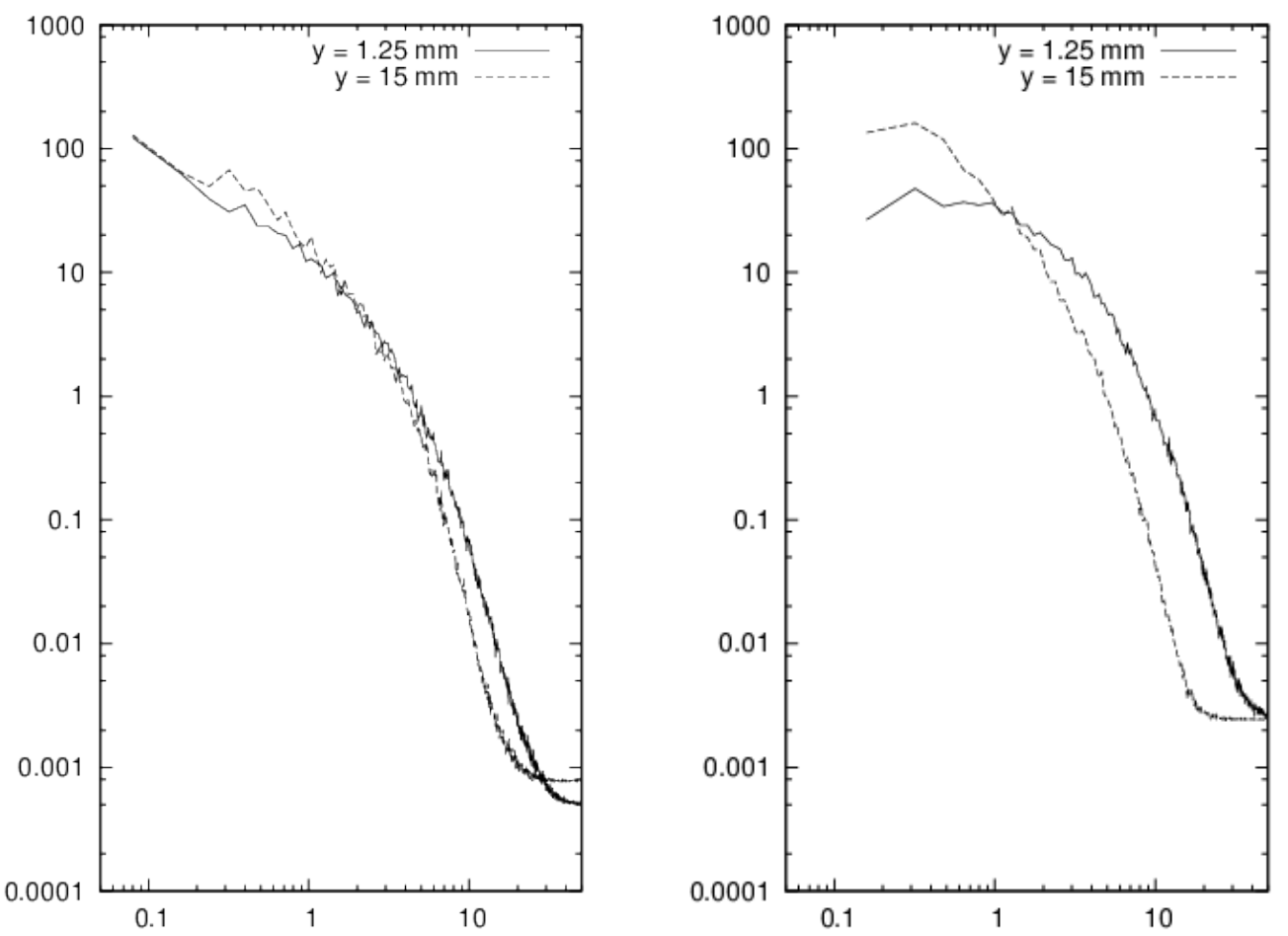

(a) Streamwise direction $\left(k_{x}\right)$ at $\mathrm{y}=1.25 \mathrm{~mm}$ and (b) Spanwise direction $\left(k_{z}\right)$ at $\mathrm{y}=1.25 \mathrm{~mm}$ and $\mathrm{y}=15 \mathrm{~mm}$ $\mathrm{y}=15 \mathrm{~mm}$

FiguRE 10. One-dimensional temperature spectra at the cold side and at the middle of the channel in the streamwise and spanwise directions.

This mechanism illustrates that the mathematical analogy between the Simpson and the Van Driest transformations has a physical basis. The mean streamwise velocity is modified by the wall-normal velocity created by the temperature gradient or coming from the injection.

Figures 14, 15 and 16 plot the root-mean-square (rms) velocity fluctuations at the cold and hot walls with classic and semi-local scalings in the streamwise, normal and respectively spanwise directions. The semi-local scaling suggested in Huang et al. (1995) is performed using the following equations

$$
u_{\tau}^{\star}=\sqrt{\frac{\tau_{w}}{<\rho>(y)}} \quad ; \quad y^{\star}=\frac{y u_{\tau}^{\star}}{<\nu>(y)}
$$

where $<.>$ is a Reynolds average operator. In the 3 directions, the root-mean-square velocity fluctuations are very different at the hot and cold walls: the temperature gradient creates an important asymmetry. The root-mean-square velocity fluctuations are always more important at the cold wall that is the most turbulent side. Moreover, the velocity fluctuation peak location of the hot side is always closer to the wall than the one of the cold side when classic scaling is used. Both the level and the peak location of the incompressible rms velocity fluctuations are included between the hot and the cold rms velocity fluctuations. The semi-local scaling allows to reduce significantly the differences between the hot and the cold walls. In particular, with the semi-local scaling the peak location of the hot. Furthermore, with the semi-local scaling, the rms velocity fluctuations 


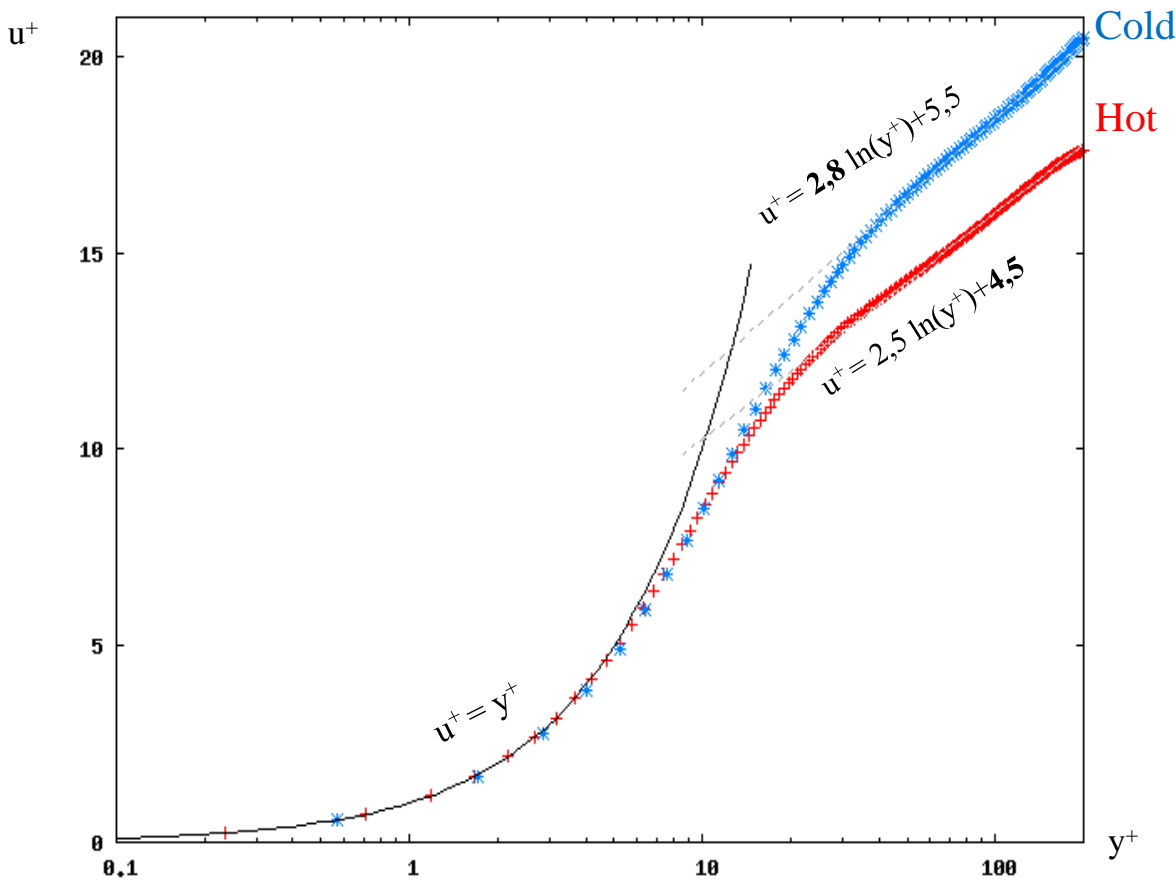

FiguRE 11. Non transformed mean velocity profile in wall units at the cold and hot walls.

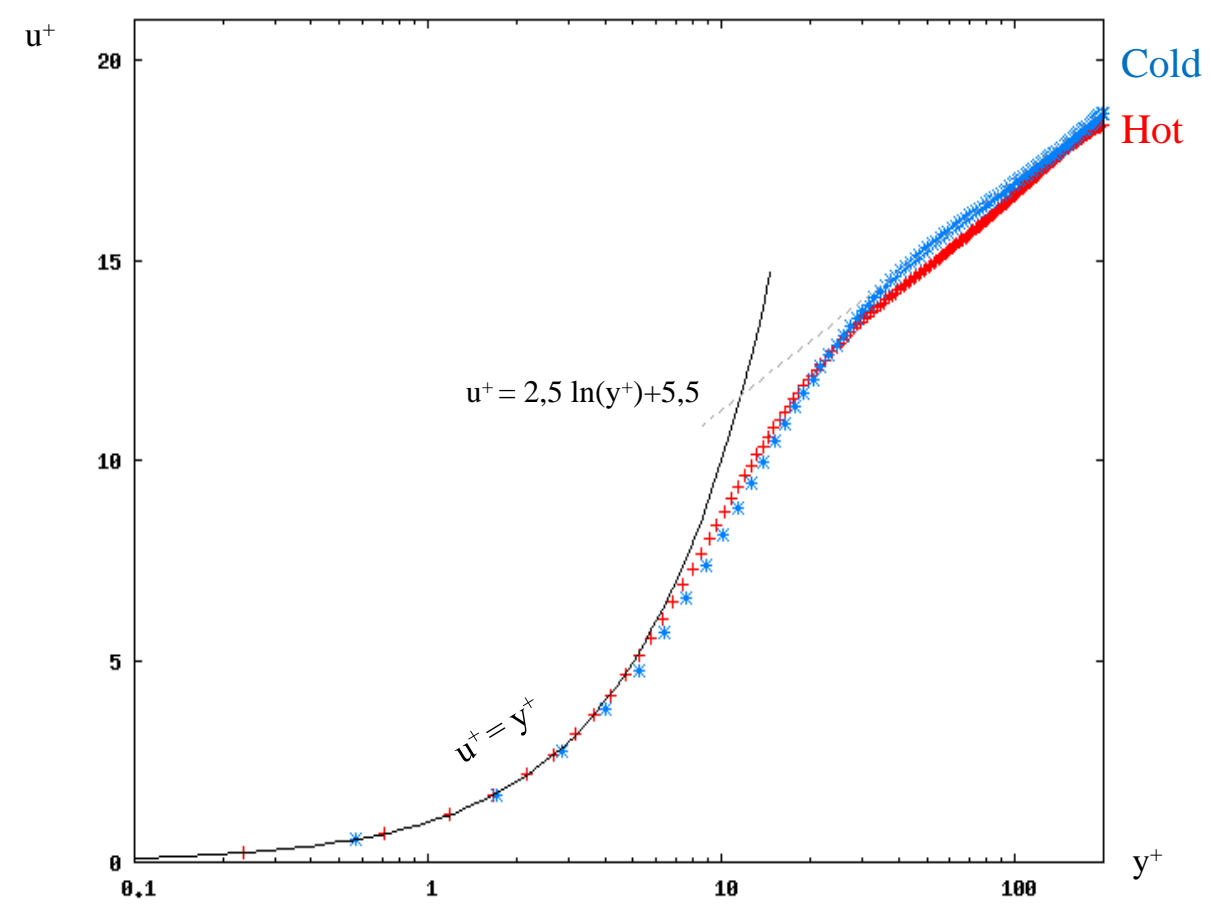

Figure 12. Transformed (Van Driest (1951)) mean velocity profiles in wall units at the cold and hot walls. 


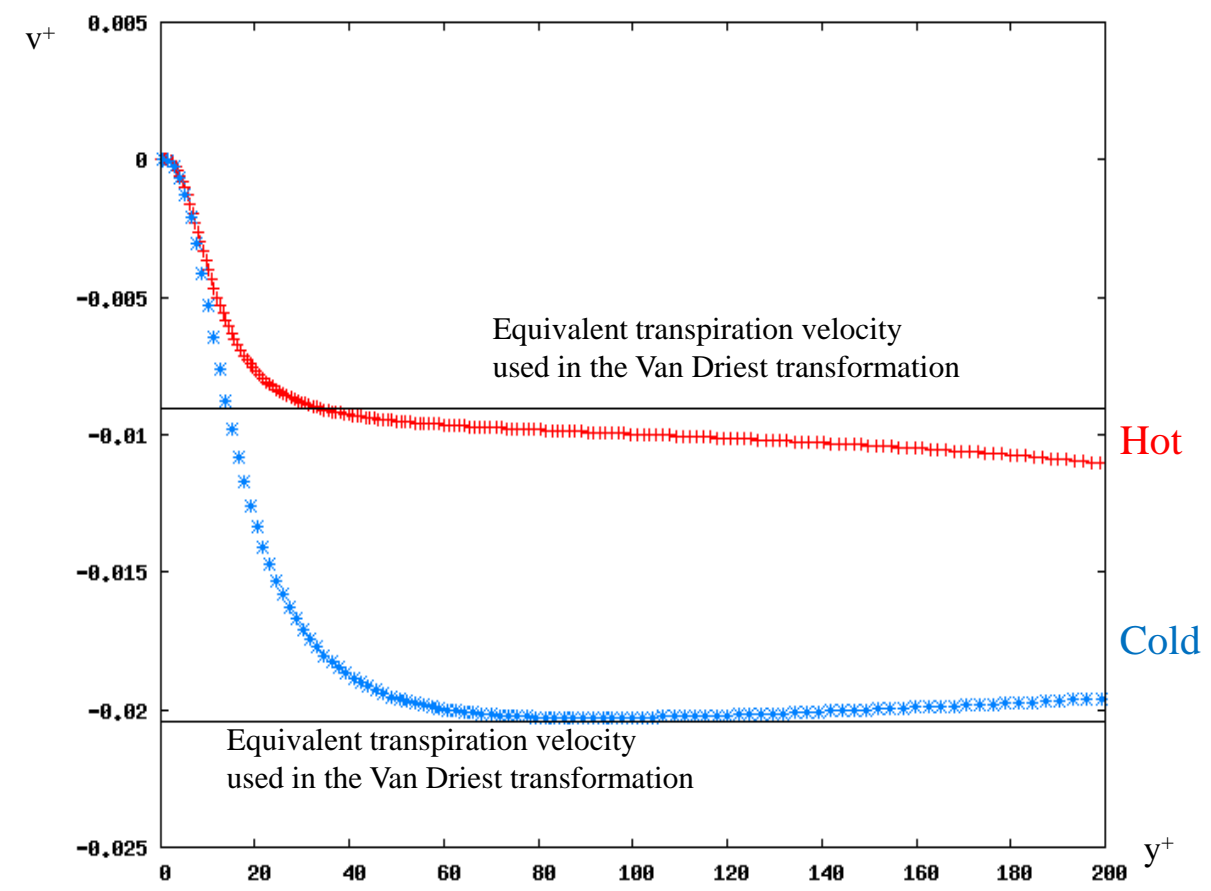

Figure 13. Comparison in wall units between the wall-normal velocity and the equivalent transpiration velocity used in the Van Driest transformation at the cold and hot walls.

of the cold side are in very good agreement with the incompressible case. However, even with this scaling, the fluctuation profiles of the hot and cold sides do not collapse. This suggests that the between the turbulence and the temperature gradient is more complex than a simple effect of the fluid property variations. can note that the most important differences between hot and cold sides are in the spanwise direction ; then, get the normal direction and finally the streamwise direction. It seems that the asymmetry increases when the mean velocity decreases (the minimum of asymmetry is obtained in the streamwise direction).

Figure 17 shows the correlations between the streamwise velocity and the wall-normal velocity. With the classic scaling, the profiles of the hot and cold sides are very different. The correlations of the cold side are much bigger than those obtained at the hot side. With the semi-local scaling, the profiles of the cold and hot sides collapse until $y^{*}=30$. However, the asymmetry remains important for $y^{*}>50$. The cold side profile matches well with the incompressible case. Figure 18 plots the correlation coefficient of the streamwise and wall-normal velocity fluctuations at the cold and hot walls:

$$
R_{u} v=-\frac{<u^{\prime} v^{\prime}>}{U_{r m s} V_{r m s}}
$$

Again, we observe an asymmetry between the hot and cold side profiles. For $y / h<0.1$, the cold side and the incompressible case plots are in perfect agreement. However, for $y / h>0.1$, none of the hot and cold side profiles match with the incompressible case. Moreover, the correlation coefficient of the hot side is bigger than the one of the cold side contrary to the velocity fluctuation profiles. It suggests that there is a turbulence production phenomena at the hot side due to the temperature gradient. This result 

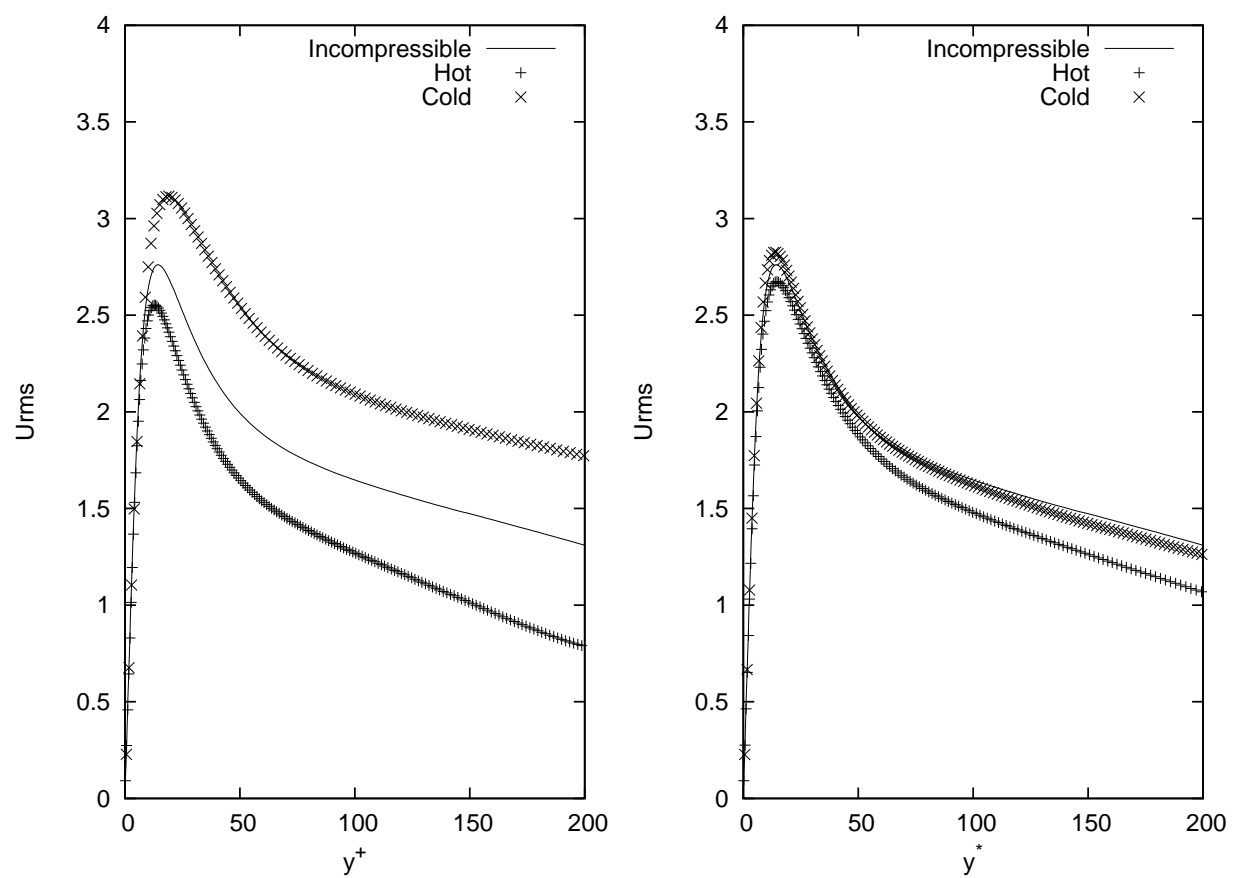

FIGURE 14. Root-mean-square velocity fluctuations in the streamwise direction at the cold and hot walls. Left side: classic scaling, right side: semi-local scaling.

is coherent with those of Serra et al. (2012a) who compare the fluctuation profiles of isothermal and anisothermal flows at equivalent Reynolds number. The maximum of the hot side correlation coefficient is located further from the wall than the cold one. It implies that, for the cold side, the local peak has the same location than the maximum streamwise velocity fluctuation as observed by Kim et al. (1987). Nevertheless, for the hot side, these two peaks have a different location.

In summary, the fluid property variations explain well the modifications of the mean velocity but do not explain the modification of the velocity fluctuations. The Van Driest transformation allows the hot and cold mean velocity profiles to collapse. Neither the classic nor the semi-local scaling the hot and cold velocity fluctuations. The same trend was observed by Nicoud (1998) for a mean turbulent Reynolds number equal to 180.

\subsection{Temperature}

In figures 20-24 presented in this section, the graphs for the "incompressible case" correspond to fluid properties independent of temperature. For density, it is realized by a very small temperature ratio $T_{r}=1+\epsilon$ with $\epsilon=0.01$. The non-dimensionalized mean temperature is defined by

$$
T^{+}=\frac{T_{w}-<T>}{T_{\tau}}
$$

where $T_{w}$ is the temperature at the wall, $<.>$ is a Reynolds average operator, $T_{\tau}$ is the friction temperature

$$
T_{\tau}=\frac{Q_{w}}{\rho_{w} C_{p} u_{\tau}}
$$



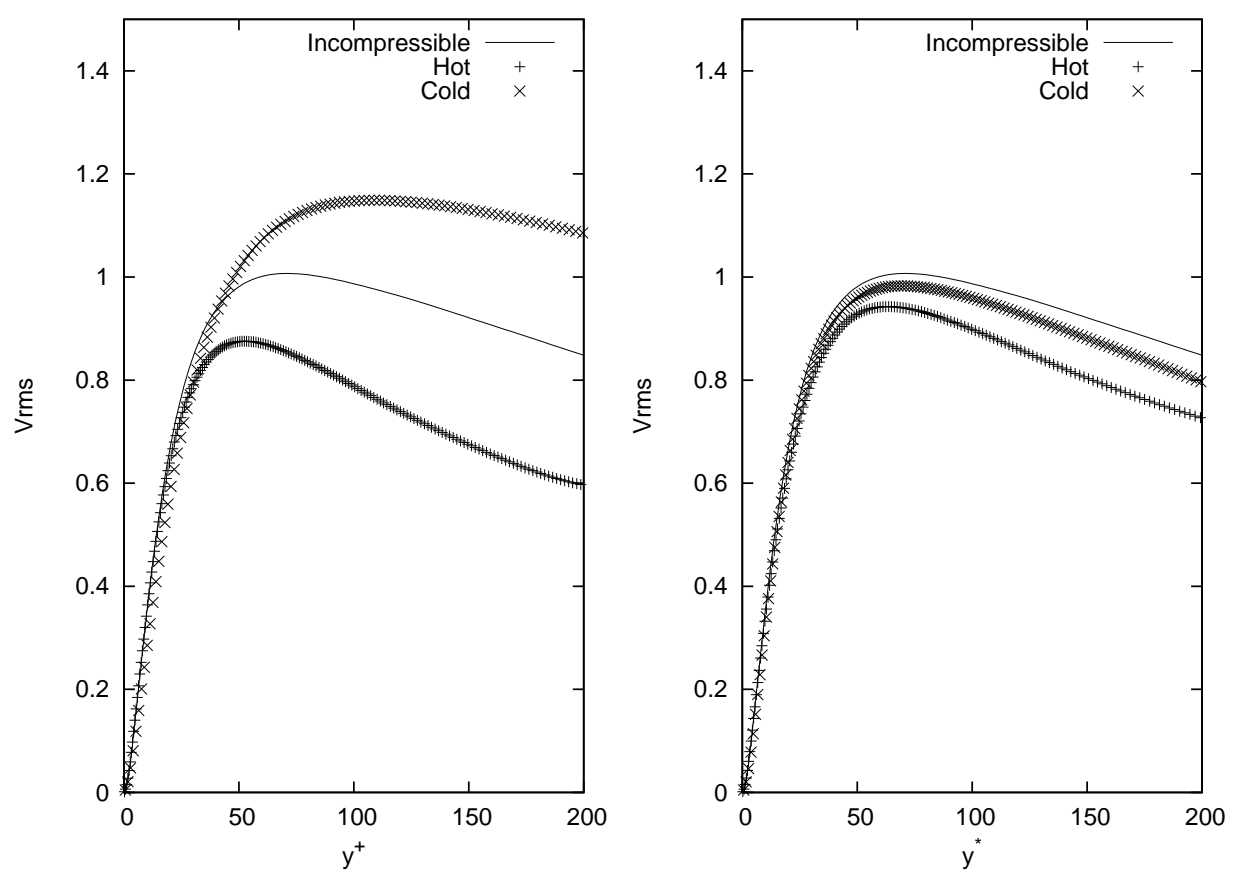

FIGURE 15. Root-mean-square velocity fluctuations in the wall-normal direction at the cold and hot walls. Left side: classic scaling, right side: semi-local scaling.
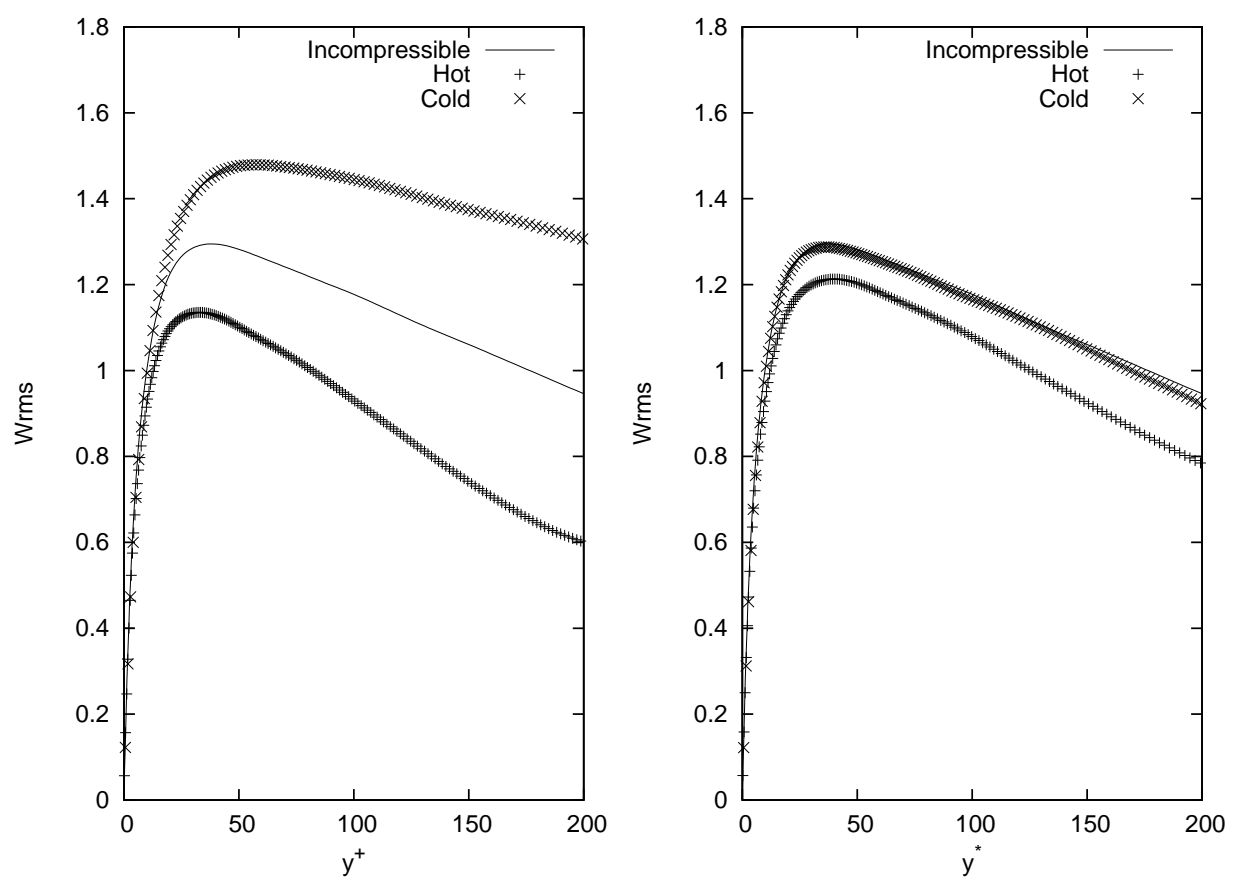

FiguRE 16. Root-mean-square velocity fluctuations in the spanwise direction at the cold and hot walls. Left side: classic scaling, right side: semi-local scaling. 

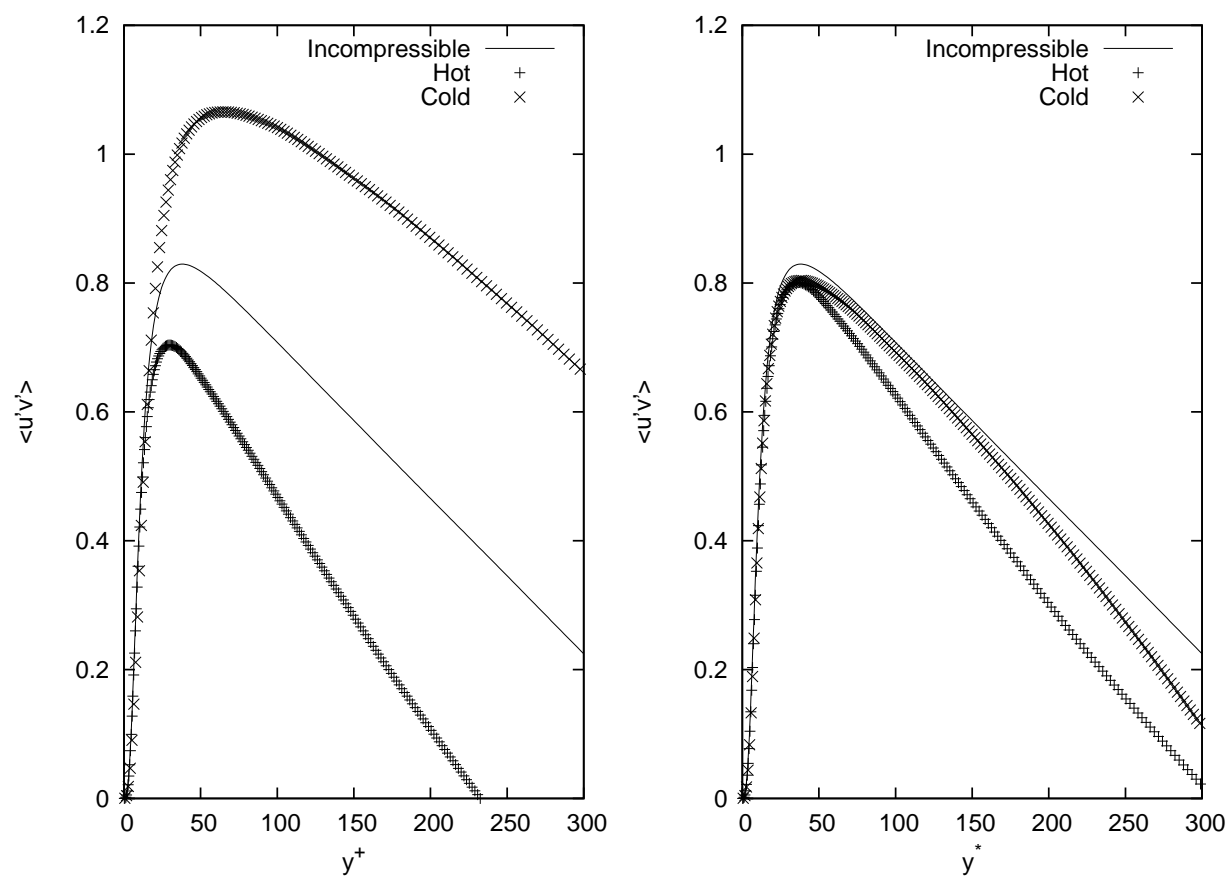

FiguRE 17. Correlation of the streamwise and wall-normal velocity fluctuations at the cold and hot walls. Left side: classic scaling, right side: semi-local scaling.

with $Q_{w}$ the heat flux at the wall. For the semi-local scaling suggested by Huang et al. (1995), the previous friction temperature is replaced by:

$$
T_{\tau}^{\star}=\frac{Q_{w}}{<\rho>(y) C_{p} u_{\tau}^{*}}
$$

The mean temperature profiles at the hot and cold walls are plotted figure 19 using the classic and the semi-local scalings. The mean profiles of the hot and cold sides seem to roughly collapse with the classic scaling. However, one can note that the curves realised with the classic scaling do not match with the standard law suggested by Kader (1981). In the logarithmic region, the Kader's law is:

$$
\begin{aligned}
T^{+} & =2.12 \ln \left(y^{+}\right)+f(P r) \\
f(P r) & =\left(3.85 \operatorname{Pr}^{(1 / 3)}-1.3\right)^{2}+2.12 \ln (\operatorname{Pr})
\end{aligned}
$$

The semi-local scaling allows the mean temperature profile of the cold side to be in perfect agreement with Kader's law. At the opposite, the profile of the hot side does not collapse with this law. Nicoud (1998) observed the same trend for a mean turbulent Reynolds number equal to 180. As he explained, in this case, it is due to a low Reynolds number effect: at the hot wall, the Peclet number $\left(P e_{2, \text { Nicoud }}=62\right)$ is too low for seeing the logarithmic region before the inflexion point. In the present case, this explanation is no valid. Indeed, as the turbulent Reynolds number $\left(R e_{\tau m}=400\right)$ is higher, the Peclet number of the present DNS at the hot wall $\left(P e_{2}=166\right) \dagger$ is bigger than the Peclet number at the cold wall $\left(P e_{1, N i c o u d}=152\right)$ in the DNS of Nicoud (1998). Consequently, a logarithmic region exists at the hot side but the mean temperature profile does not

$\dagger$ The Peclet number of the present DNS at the cold wall is $P e_{1}=401$. 


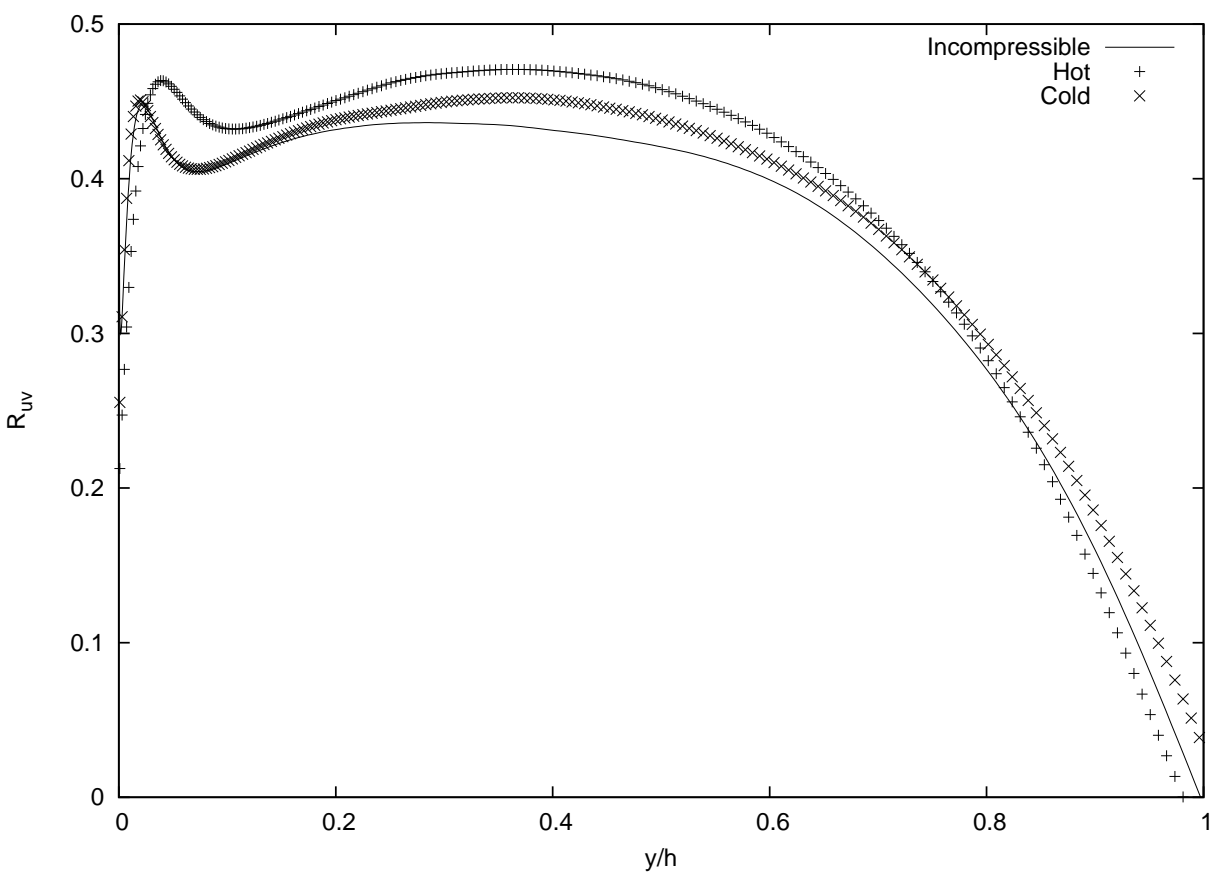

FIGURE 18. Correlation coefficient of the streamwise and wall-normal velocity fluctuations at the cold and hot walls.

match with Kader's law. Again, it suggests that the asymmetries created by the high temperature gradient cannot be reduced to a simple effect of the fluid property variations. The strong coupling between turbulence and temperature gradient modifies the standard law. Figure 20 presents the root-mean-square temperature fluctuations at the hot and cold sides. The profiles do not collapse neither for the classic nor for the semi-local scalings. Contrary to the rms velocity fluctuations, the semi-local scaling does not allow the rms temperature fluctuations of the cold side to be in very good agreement with the incompressible case. In particular, the rms temperature plots for the incompressible case and the cold wall deviate considerably for $y^{+}>50$. Contrary to the rms velocity fluctuations, the semi-local scaling allows the rms temperature of the hot side to be higher than the one of the cold side which is physically coherent. Indeed, .

Gaviglio (1987) shows that temperature and velocity fluctuations are highly correlated within large coherent structures. Defining the characteristic length as $l_{u}=\frac{U_{r m s}}{\partial<u>/ \partial y}$ for the velocity and as $l_{T}=\frac{T_{r m s}}{\partial<T>/ \partial y}$ for the temperature, he assumes that $l_{u} \propto l_{T}$ and derives:

$$
\frac{T_{r m s}}{U_{r m s}} \frac{\left|\frac{\partial<u>}{\partial y}\right|}{\left|\frac{\partial<T>}{\partial y}\right|}=R_{0}
$$

Figure 21(a) illustrates this hypothesis that appears to be a reasonable representation of the present results. One can note that $R_{0}=\frac{1}{P r_{t}}$ with $P r_{t} \approx 0.9$ proposed by Huang et al. (1995) is a good choice. The turbulent Prandtl number is calculated from the DNS 

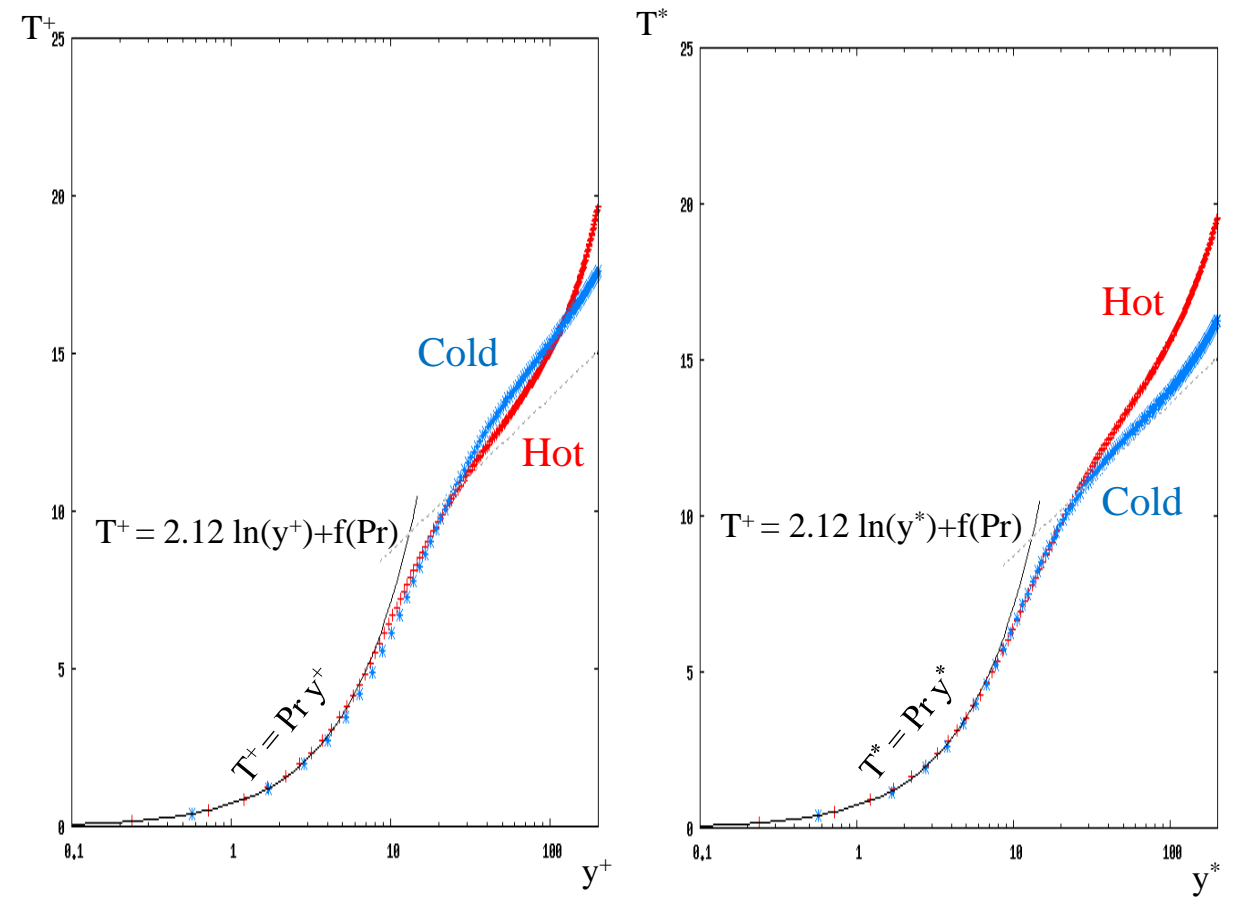

FiguRE 19. Mean temperature profile in wall units at the cold and hot walls. Left side: classic scaling, right side: semi-local scaling.
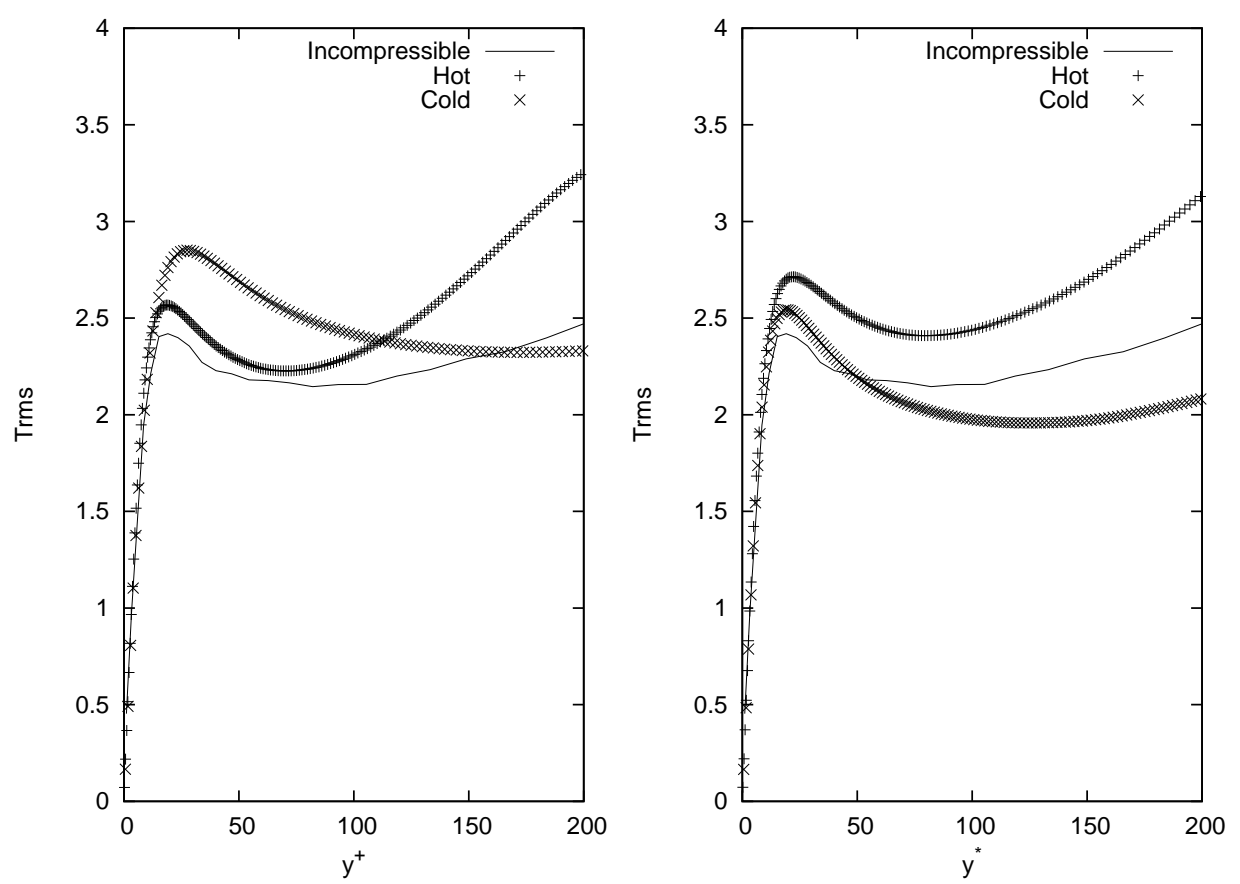

FigURE 20. Root-mean-square temperature fluctuations in wall units at the cold and hot walls. Left side: classic scaling, right side: semi-local scaling. 
results using:

$$
P r_{t}=\frac{\nu_{t}}{\alpha_{t}}=\frac{<u^{\prime} v^{\prime}>}{<v^{\prime} T^{\prime}>} \frac{\partial<T>/ \partial y}{\partial<u>/ \partial y}
$$

The profiles are given by figure 21(b). The turbulent Prandtl numbers of the cold and hot sides of the channel look more similar than for smaller turbulent Reynolds number $\left(R e_{\tau m}=180\right)$. In particular, the absence of peak at the wall of the hot side showed by Nicoud (1998) for $R e_{\tau m}=180$ seems to be a low Reynolds effect. The present results with $R e_{\tau m}=400$ show a peak at the wall of the cold and hot sides. However, the profiles of the hot and cold sides have an opposite behavior for $\frac{y}{h}>0.4$. The peak at the hot side is due to the asymmetry created by the temperature gradient. The asymmetry has for consequence that the location of sign change is different for the velocity-velocity correlation $\left\langle u^{\prime} v^{\prime}>\right.$ and for the mean velocity gradient $\partial<u>/ \partial y$.

Figures 22 and 23 give the correlations of the streamwise velocity respectively the wall-normal velocity and the temperature. With the classic scaling, the profiles of the hot and cold sides are very different. The correlations obtained at the cold side are much higher than the one of the hot side. With the semi-local scaling, the correlations of the streamwise velocity and temperature are almost identical for the hot and cold sides. For the correlations of the wall-normal velocity and temperature, the asymmetry remains even with the semi-local scaling. More interesting, the correlations at the hot side are higher than at the cold side. Figure 24 plots the coefficient correlation of the velocity and temperature fluctuations for the streamwise velocity

$$
R_{u T}=\frac{<u^{\prime} T^{\prime}>}{U_{r m s} T_{r m s}}
$$

and the wall-normal velocity:

$$
R_{v T}=\frac{<v^{\prime} T^{\prime}>}{V_{r m s} T_{r m s}}
$$

The maximum of $R_{u T}$ and $R_{v T}$ is located further from the wall at the hot side. The same trend is obtained by Nicoud (1998). In agreement with Nicoud (1998), we find also that the profile of $R_{u T}$ is higher at the hot side. However, the differences are lighter in our case. Indeed, at $y / h=0.5$, we have $R_{u T, 1} \approx R_{u T, 1, \text { Nicoud }} \approx 0.5$ compared to $R_{u T, 2} \approx 0.6<R_{u T, 2, N i c o u d} \approx 0.8$. Consequently, it seems that the asymmetry of the coefficient correlation of streamwise velocity and temperature fluctuations is linked to the turbulent intensity.

Serra et al. (2012c)Serra et al. (2012c) Zhou (1995)

\section{Conclusion}

Direct numerical simulations of a turbulent channel flow submitted to a high temperature gradient are carried out with 470 millions $(1537 \times 397 \times 769)$ mesh points at a Reynolds number of 6400 . The computed results of the cold and hot walls are compared. The nondimensionnalization that takes into account the variations of the fluid properties damps the asymmetry between the cold and the hot walls. In particular, the Van Driest nondimensionnalization allows to recover the classical log law for the mean velocity. We show that the mean velocity normal to the wall and due to temperature gradient creates the asymmetry. It explains that the mathematical analogy between the Van Driest and the Simpson transformations has a physical basis: the wall-normal velocity created respectively by the temperature gradient or the injection. The Kader's law is not recovered for the mean temperature profile. It illustrates that the coupling between the turbulence and 


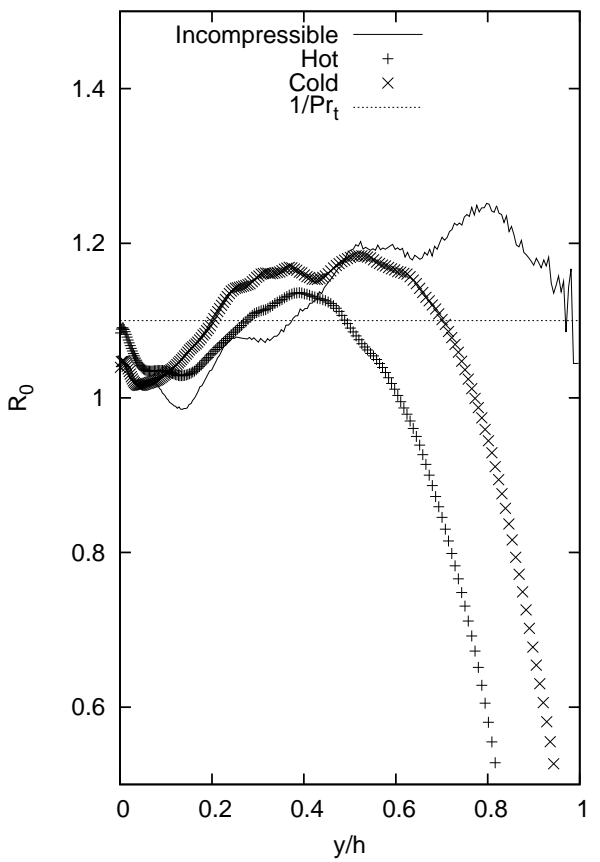

(a)

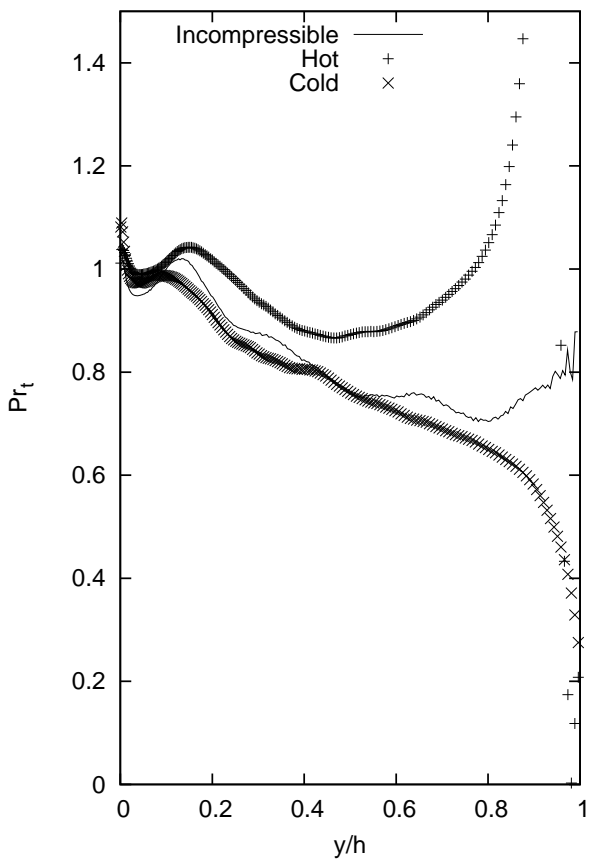

(b)

Figure 21. $R_{0}$ coefficient (left) and turbulent Prandtl number (right) from DNS at the hot and cold walls.
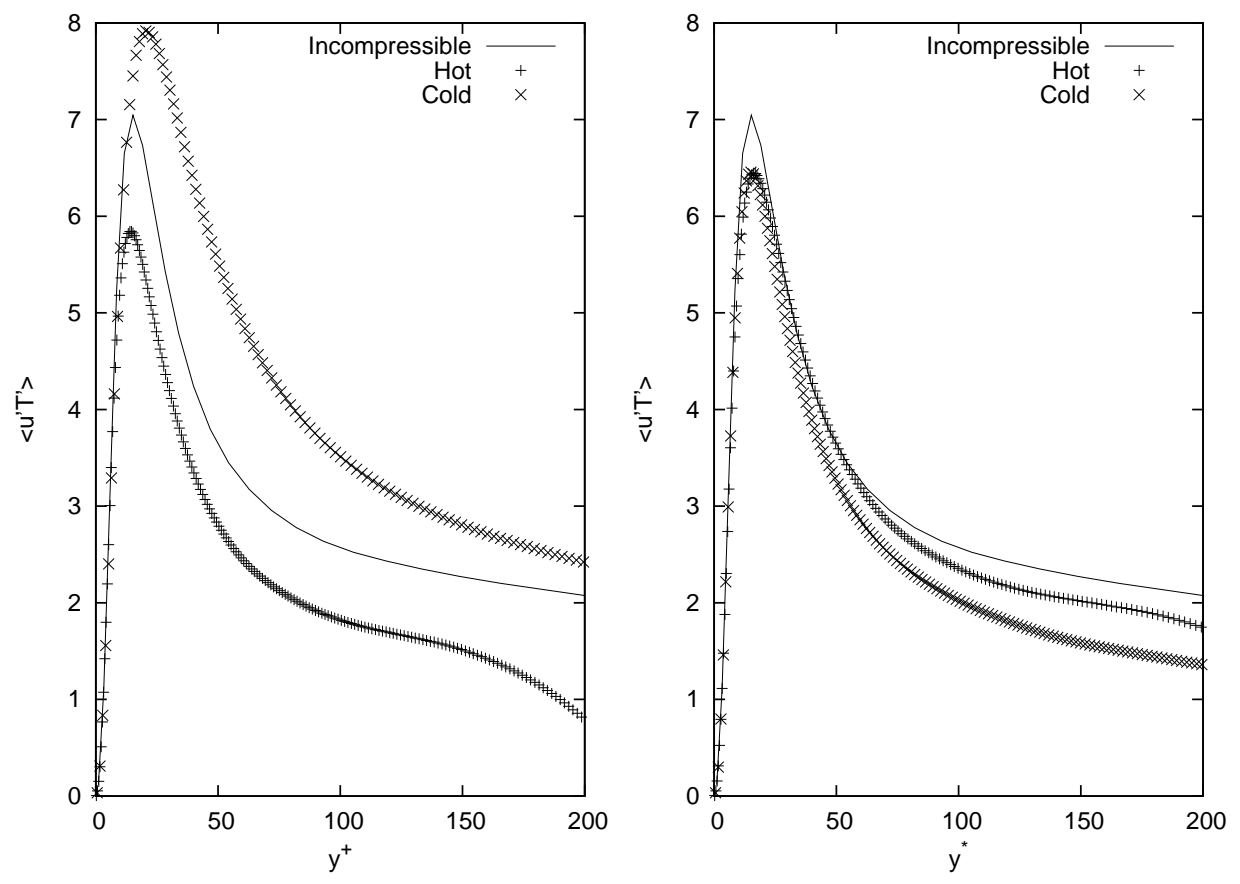

Figure 22. Correlation of the streamwise velocity and temperature fluctuations at the cold and hot walls. Left side: classic scaling, right side: semi-local scaling. 

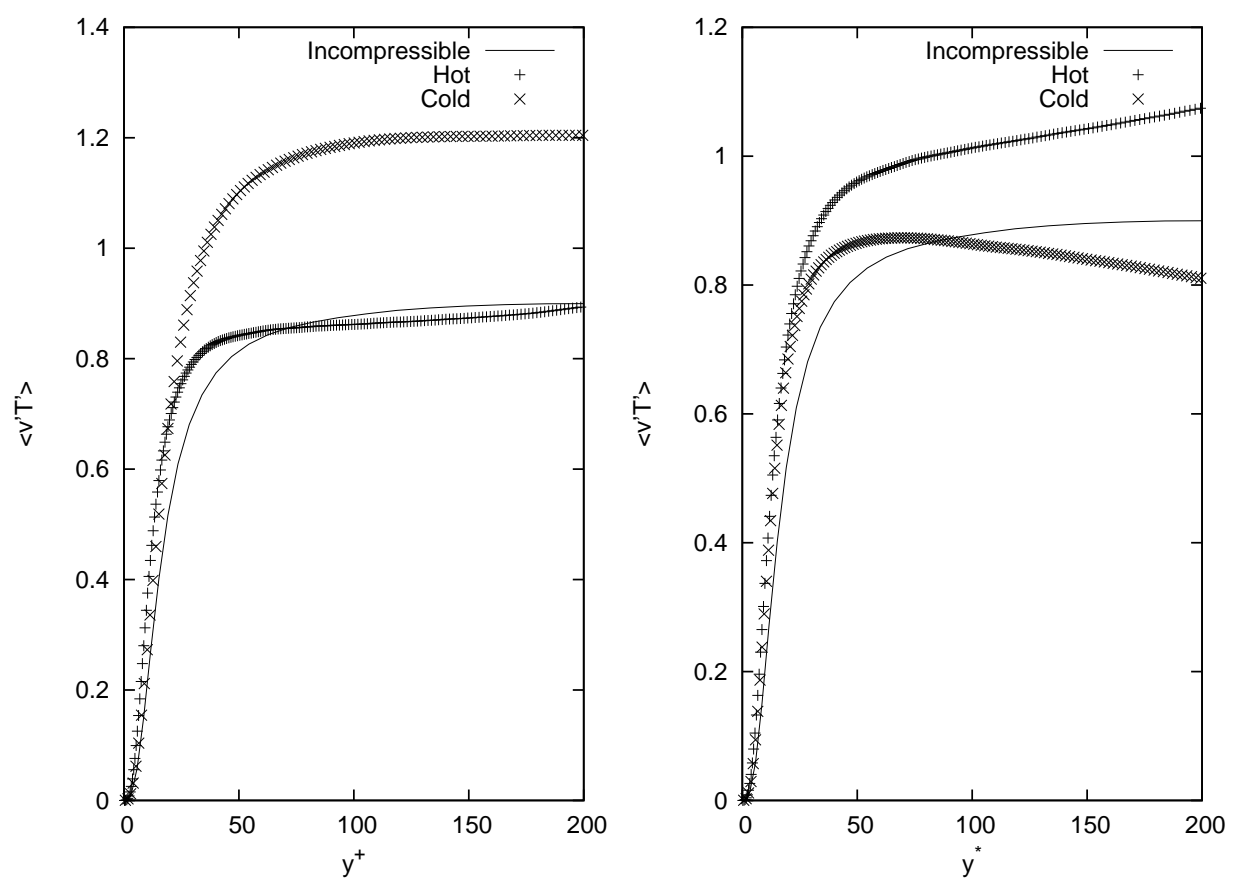

FIGURE 23. Correlation of the wall-normal velocity and temperature fluctuations at the cold and hot walls. Left side: classic scaling, right side: semi-local scaling.
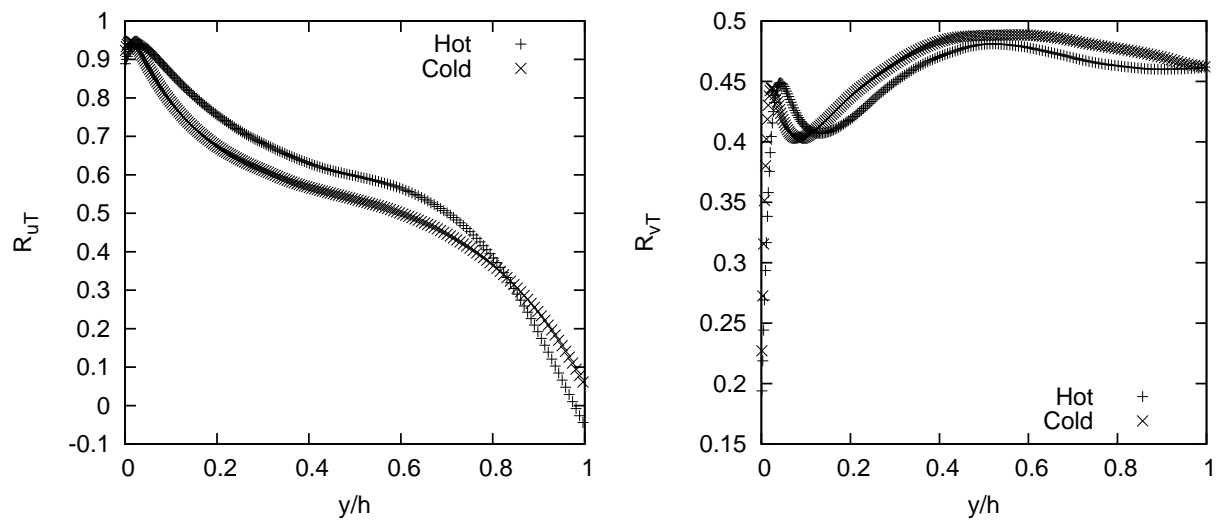

FIGURE 24. Coefficient correlation of the velocity and temperature fluctuations at the cold and hot walls. Left side: streamwise velocity, right side: wall-normal velocity.

the temperature gradient is more complex than the mean variations of fluid properties. The rms velocity fluctuations are higher at the cold side and, at the opposite, the rms temperature fluctuations are higher at the hot side. The following physical mechanism can explain this behavior:

- the increase of viscosity at the hot side tends to dissipate turbulent kinetic energy (rms velocity fluctuations are higher at the cold side),

- consequently the turbulent mixing is smaller at hot side, the temperature is less homogeneous and rms temperature fluctuations are higher. 
It is worth noting that one observes the same behavior at the channel flow center where rms velocity fluctuations are small and rms temperature fluctuations are high. It is also important to precise that the high rms temperature fluctuations at the hot side produces turbulent kinetic energy thanks to dilatational effect. This production process is in competition with the viscous effect (dissipation mechanism). This behavior has also been shown in a previous work where we compare the rms velocity profiles of isothermal and anisothermal case at equivalent Reynolds number (see Serra et al. $(2012 b)$ ). The correlations (velocity-velocity, temperature-velocity) of the hot and cold walls do not collapse. Finally, we showed and identified the low Reynolds effects and the influence of the temperature gradients on the turbulent statistic quantities.

\section{Acknowledgment}

The authors would like to acknowledge the support of the CINES (France), which provided computer resources to carry out the simulations. They are also thankful to the CEA (France Atomic Agency), for their support with the Trio_U code.

\section{REFERENCES}

Brillant, G., Husson, S. \& Bataille, F. 2005 Large eddy simulations of heat and mass transfers in case of non isothermal blowing. In Engineering Turbulence Modelling and Experiments 6 (ed. W. Rodi \& M. Mulas), pp. 751-760. ERCOFTAC, Sardinia, Italy.

Calvin, C., Cueto, O. \& Emonot, P. 2002 An object-oriented approach to the design of fluid mechanics software. Mathematical modelling and numerical analysis 36 (5), 907-921.

Châtelain, A., Ducros, F. \& MÉtais, O. 2004 LES of turbulent heat transfer: proper convection numerical schemes for temperature transport. International Journal for Numerical Methods in Fluids 44 (9), 1017-1044.

Coleman, G. N., J., Kim. \& Moser, R. D. 1995 A numerical study of turbulent supersonic isothermal-wall channel flow. Journal of Fluid Mechanics 305, 159-183.

Daguenet-Frick, Xavier, Foucaut, Jean-Marc, Coudert, Sebastien, Toutant, Adrien \& Olalde, Gabriel 2012 Experimental analysis of the turbulent flow behavior of a textured surface proposed for asymmetric heat exchangers. Flow, Turbulence and Combustion 89, 149-169.

Debusschere, B. \& Rutland, C. J. 2004 Turbulent scalar transport mecanisms in plane channel and couette flows. International Journal of Heat and Mass Transfer 47, 17711781.

Elmo, M. \& Cioni, O. 2003 Low mach number model for compressible flows and application to htr. Nuclear Engineering and Design 222, 117-124.

Gaviglio, J. 1987 Reynolds analogies and experimental study of heat transfer in the supersonic boundary layer. International Journal Heat and Mass Transfert 30 (5), 911-926.

Hinze, J. 1975 Introduction to turbulence modeling. Mc Graw-Hill 2.

Huang, P. G., Coleman, G. N. \& Bradshaw, P. 1995 Compressible turbulent channel flows: DNS results and modelling. Journal of Fluid Mechanics 305, 185-218.

KADER, B. A. 1981 Temperature and concentration profiles in fully turbulent boundary layers. Int. J. Heat Mass Transfer 24, 1541-1544.

KawAmuRA, H. 2008 DNS database of wall turbulence and heat transfer. Kawamura lab (http://murasun.me.noda.tus.ac.jp/turbulence/index.html).

Kawamura, H., Abe, H. \& Matsuo, Y. 1999 DNS of turbulent heat transfer in channel flow with respect to Reynolds and Prandtl number effect. International Journal of Heat and Fluid Flow 20, 196-207.

Kawamura, H., Abe, H. \& Shingai, K. 2000 DNS of turbulence and heat transport in a channel flow with different Reynolds and Prandtl numbers and boundary conditions. In 3rd International Symposium on Turbulence, Heat and Mass Transfer, Aichi Shuppan, Tokyo (ed. Y. Nagano, K. Hanjalic \& T. Tsuji), pp. 15-32. 
Kim, J., Moin, P. \& Moser, R. 1987 Turbulence statistics in fully developed channel flow at lox reynolds number. Journal of Fluid Mechanics 177, 133-166.

Lagha, M., Kim, J., Eldredge, J. D. \& Zhong, X. 2011 A numerical study of compressible turbulent boundary layers. Physics of Fluids 23 (015106).

Lele, Sanjiva K. 1992 Compact finite difference schemes with spectral-like resolution. Journal of Computational Physics 103 (1), 16 - 42.

Lessani, B. \& PAPAlEXANDRIS, M. V. 2006 Time-accurate calculation of variable density flows with strong temperature gradients and combustion. Journal of Computational Physics 212, $218-246$.

Lessani, B. \& Papalexandris, M. V. 2007 Numerical study of turbulent channel flow with strong temperature gradients. International Journal of Numerical Methods for Heat $\&$ Fluid Flow 18, 545-556.

Mahalov, A., Nicolaenko, B. \& Zhou, Y. 1998 Energy spectra of strongly stratified and rotating turbulence. Physical Review E 57 (5), 6187-6190.

Majda, A. \& Sethian, J.A. 1985 The derivation and numerical solution of the equations for zero mach number combustion. Combust. Sci. Tech. 42, 185-205.

Marusic, I., McKeon, B. J., Monkewitz, P. A., Nagib, H. M., Smits, A. J. \& R., SreeniVASAN K. 2011 Wall-bounded turbulent flows at high reynolds numbers: Recent advances and key issues. Physics of Fluids 23 (065103).

Morinishi, Y., Tamano, S. \& Nakabayashi, K. 2004 Direct numerical simulation of compressible turbulent channel flow between adiabatic and isothermal walls. Journal of Fluid Mechanics 502, 273-308.

Morinishi, Youhei, Tamano, Shinji \& Nakamura, Eisuke 2007 New scaling of turbulence statistics for incompressible thermal channel flow with different total heat flux gradients. International Journal of Heat and Mass Transfer 50 (9-10), 1781 - 1789.

Moser, R. D., Kim, J. \& Mansour, N. N. 1999 Direct numerical simulation of turbulent channel flow up to $R e_{\tau}=590$. Physics of Fluids, database http://turbulence.ices.utexas.edu/MKM_1999.html 11 (4), 943-945.

Nicoud, F. \& Bradshaw, P. 2000 A velocity transformation for heat and mass transfer. Physics of Fluids 12 (1), 237-238.

NiCOUD, F. C. 1998 Numerical study of a channel flow with variable properties. Center for Turbulent Research, Annual Research Briefs pp. 289-309.

PAOlucCI, S. 1982 On the filtering of sound from the navier-stockes equations. Tech. Rep. SAND82-8257. SANDIA National Labs., Livermore, CA (USA).

RichARDSON, L. F. 1911 The approximate arithmetical solution by finite differences of physical problems involving differential equations, with an application to the stresses in a masonry dam. Philosophical Transactions of the Royal Society of London. Series A, Containing Papers of a Mathematical or Physical Character 210 (459-470), 307-357.

Serra, Sylvain, Toutant, Adrien \& Bataille, Franoise $2012 a$ Thermal large eddy simulation in a very simplified geometry of a solar receiver. Heat Transfer Engineering 33 (6), $505-524$.

Serra, Sylvain, Toutant, Adrien, Bataille, Franoise \& Zhou, Ye $2012 b$ Hightemperature gradient effect on a turbulent channel flow using thermal large-eddy simulation in physical and spectral spaces. Journal of Turbulence 13 (49), 1-25.

Serra, Sylvain, Toutant, Adrien, Bataille, Franoise \& Zhou, Ye $2012 c$ Turbulent kinetic energy spectrum in very anisothermal flows. Physics Letters A 376 (45), 3177 - 3184.

Simpson, R. L. 1970 Characteristics of turbulent boundary layers at low reynolds numbers with and without transpiration. Journal of Fluid Mechanics 42, 769-802.

Spina, E. F., Smits, A. J. \& Robinson, S. K. 1994 The physics of supersonic turbulent boundary layers. Ann. Rev. Fluid Mech. 26, 287-319.

TAmano, S. \& Morinishi, Y. 2006 Effect of different thermal wall boundary conditions on compressible turbulent channel flow at m=1.5. Journal of Fluid Mechanics 548, 361-373.

VAN DRIEst, E. R. 1951 Turbulent boundary layer in compressible fluids. Journal of Aero. Sciences 18 (3), 145-160.

WANG, W.-P. \& Pletcher, R. H. 1996 On the large eddy simulation of a turbulent channel flow with significant heat transfer. Physics of Fluids $\mathbf{8}$ (12), 3354-3366.

Wei, Liang \& Pollard, Andrew 2011 Direct numerical simulation of compressible turbulent 
channel flows using the discontinuous galerkin method. Computers and Fluids 47 (1), 85 -100 .

Wu, Xiaohua \& Moin, Parviz 2010 Transitional and turbulent boundary layer with heat transfer. Physics of Fluids 22 (8), 085105.

YE \& ZHOU 2010 Renormalization group theory for fluid and plasma turbulence. Physics Reports 488 (1), $1-49$.

Zhou, Ye 1995 A phenomenological treatment of rotating turbulence. Physics of Fluids 7 (8), 2092-2094.

Zhou, Y. \& Oughton, S. 2011 Nonlocality and the critical reynolds numbers of the minimum state magnetohydrodynamic turbulence. Physics of Plasmas 18 (7). 